\title{
Three $s$-wave interacting fermions under anisotropic harmonic confinement: Dimensional crossover of energetics and virial coefficients
}

\author{
Seyed Ebrahim Gharashi, ${ }^{1}$ K. M. Daily, ${ }^{1}$ and D. Blume ${ }^{1,2}$ \\ ${ }^{1}$ Department of Physics and Astronomy, Washington State University, Pullman, Washington 99164-2814, USA \\ ${ }^{2}$ ITAMP, Harvard-Smithsonian Center for Astrophysics, \\ 60 Garden Street, Cambridge, Massachusetts 02138, USA
}

(Dated: June 18, 2021)

\begin{abstract}
We present essentially exact solutions of the Schrödinger equation for three fermions in two different spin states with zero-range $s$-wave interactions under harmonic confinement. Our approach covers spherically symmetric, strictly two-dimensional, strictly one-dimensional, cigar-shaped, and pancake-shaped traps. In particular, we discuss the transition from quasi-one-dimensional to strictly one-dimensional and from quasi-two-dimensional to strictly two-dimensional geometries. We determine and interpret the eigenenergies of the system as a function of the trap geometry and the strength of the zero-range interactions. The eigenenergies are used to investigate the dependence of the second- and third-order virial coefficients, which play an important role in the virial expansion of the thermodynamic potential, on the geometry of the trap. We show that the second- and third-order virial coefficients for anisotropic confinement geometries are, for experimentally relevant temperatures, very well approximated by those for the spherically symmetric confinement for all $s$-wave scattering lengths.

PACS numbers:
\end{abstract}

\section{INTRODUCTION}

There has been extensive interest in ultracold atom physics in the last decade [1-3]. Ultracold atomic bosonic and fermionic gases are realized experimentally under varying external confinements. In these experiments, the number of particles and the scattering length of the two-body interactions are tunable [4]. Although the complete energy spectrum of the many-body system cannot, in general, be obtained from first principles, the energy spectra of selected few-body systems can, in some cases, be determined within a microscopic quantum mechanical framework [5- 10]. In some cases, the properties of the few-body system have then been used to predict the properties of the corresponding many-body system [11-19].

The behavior of atomic and molecular systems depends strongly on the dimensionality of the system [20 23]. In three dimensions, e.g., weakly-bound two-body $s$-wave states exist when the $s$-wave scattering length is large and positive but not when it is negative. In strictly one- and two-dimensional geometries, in contrast, $s$-wave bound states exist for all values of the $s$-wave scattering length [5].

In ultracold atomic gases, the de Broglie wavelength of the atoms is much larger than the van der Waals length that characterizes the two-body interactions. This allows one to replace the van der Waals interaction potential in free-space low-energy scattering calculations by a zero-range $s$-wave pseudopotential [24 26]. If the particles are placed in an external trap, the validity of the pseudopotential treatment (at least if implemented without accounting for the energy-dependence of the coupling strength) requires that the van der Waals length is much smaller than the characteristic trap length [27, 28]. In many cases, the use of pseudopotentials greatly simplifies the theoretical treatment. For example, the eigenequation for two particles interacting through a $s$-wave pseudopotential under harmonic confinement has been derived analytically for spherically symmetric, strictly one-dimensional, strictly twodimensional and anisotropic harmonic potentials [5] 7 .

The $s$-wave pseudopotential has also been applied successfully to a wide range of three-body problems, either in free space or under confinement [29 35]. The present paper develops an efficient numerical framework for treating the three-body system under anisotropic harmonic confinement. The developed formalism allows us to study the dependence of the three-body properties on the dimensionality of the system. We focus on fermionic systems consisting of two identical spin-up atoms and one spin-down atom. The dimensional crossover of two-component Fermi gases has attracted a great deal of interest recently [36 38]. This paper considers the three-body analog within a microscopic quantum mechanical framework. We note that our framework readily generalizes to bosonic three-body systems. The study of the dimensional crossover of bosonic systems is interesting as it allows one to study how, under experimentally realizable conditions, Efimov trimers [39] that are known to exist in three-dimensional space disappear as the confinement geometry is tuned to an effectively low-dimensional geometry [40].

This paper generalizes the methods developed in Refs. [8, 32] for three equal-mass fermions in two different pseudospin states under spherically symmetric harmonic confinement to anisotropic harmonic confinement. We develop 
an efficient and highly accurate algorithm to calculate the eigenenergies and eigenstates of the system up to relatively high energies as functions of the interaction strength and aspect ratio of the trap. Several applications are considered: (i) The BCS-BEC crossover curve is analyzed throughout the dimensional crossover. (ii) For large and small aspect ratios, the energy spectra are analyzed in terms of strictly one-dimensional and strictly two-dimensional effective three-body Hamiltonian. (iii) The second- and third-order virial coefficients are analyzed as functions of the temperature, aspect ratio and scattering length. In particular, we show that the high-temperature limit of the third-order virial coefficient $b_{3}$ at unitarity is independent of the shape of the trap in agreement with expectations derived through use of the local density approximation. For finite scattering lengths, $b_{2}$ and $b_{3}$ for anisotropic harmonic confinement are well approximated by those for isotropic harmonic confinement.

The remainder of this paper is organized as follows. Section II presents a formal solution to the problem of three $s$-wave interacting fermions confined in an axially symmetric harmonic trap. We also consider the extreme cases of strictly one-dimensional and strictly two-dimensional confinement. Sections III and IV] apply the formal solution to cigar-shaped and pancake-shaped traps, respectively. We determine a large portion of the eigenspectrum as a function of the scattering length and discuss the transition to strictly one-dimensional and strictly two-dimensional geometries. Section $[\mathrm{V}$ uses the two- and three-body eigenspectra to calculate the second- and third-order virial coefficients as a function of the temperature and the geometry of the confinement. Finally, Sec. VI concludes.

\section{FORMAL SOLUTION}

We consider a two-component Fermi gas consisting of two spin-up atoms and one spin-down atom with interspecies $s$-wave interactions under anisotropic harmonic confinement. We refer to the two spin-up atoms as particles 1 and 2 , and to the spin-down atom as particle 3 . We introduce the single-particle Hamiltonian $H_{0}\left(\mathbf{r}_{j}, \mathcal{M}\right)$ for the $j^{t h}$ particle with mass $\mathcal{M}$ under harmonic confinement,

$$
H_{0}\left(\mathbf{r}_{j}, \mathcal{M}\right)=\frac{-\hbar^{2}}{2 \mathcal{M}} \nabla_{\mathbf{r}_{j}}^{2}+\frac{1}{2} \mathcal{M}\left(\omega_{z}^{2} z_{j}^{2}+\omega_{\rho}^{2} \rho_{j}^{2}\right) .
$$

Here, $\mathbf{r}_{j}$ is measured with respect to the trap center, and in cylindrical coordinates we have $\mathbf{r}_{j}=\left(z_{j}, \rho_{j}, \phi_{j}\right)$. In Eq. (1),$\omega_{z}$ and $\omega_{\rho}$ are the angular trapping frequencies in the $z$ - and $\rho$-directions, respectively. The aspect ratio $\eta$ of the trap is defined through $\eta=\omega_{\rho} / \omega_{z}$. In this paper, we consider cigar-shaped traps with $\eta>1$ as well as pancake-shaped traps with $\eta<1$. Our three-particle Hamiltonian $H$ then reads

$$
H=\sum_{j=1}^{3} H_{0}\left(\mathbf{r}_{j}, \mathcal{M}\right)+V_{\mathrm{int}}
$$

where $V_{\text {int }}$ accounts for the interspecies $s$-wave two-body interactions,

$$
V_{\mathrm{int}}=V_{\mathrm{ps}}^{3 \mathrm{D}}\left(\mathbf{r}_{31}\right)+V_{\mathrm{ps}}^{3 \mathrm{D}}\left(\mathbf{r}_{32}\right)
$$

The regularized pseudopotential $V_{\mathrm{ps}}^{3 \mathrm{D}}$ is characterized by the three-dimensional $s$-wave scattering length $a^{3 \mathrm{D}}[24$ 26],

$$
V_{\mathrm{ps}}^{3 \mathrm{D}}\left(\mathbf{r}_{j k}\right)=\frac{4 \pi \hbar^{2} a^{3 \mathrm{D}}}{\mathcal{M}} \delta\left(\mathbf{r}_{j k}\right) \frac{\partial}{\partial r_{j k}} r_{j k},
$$

where $\mathbf{r}_{j k}=\mathbf{r}_{j}-\mathbf{r}_{k}$ and $r_{j k}=\left|\mathbf{r}_{j k}\right|$.

Since the trapping potential is quadratic, the relative and center of mass degrees of freedom separate and we rewrite the Hamiltonian $H$ in terms of the relative Hamiltonian $H_{\mathrm{rel}}$ and the center of mass Hamiltonian $H_{\mathrm{cm}}, H=H_{\mathrm{rel}}+H_{\mathrm{cm}}$. In the following, we obtain solutions to the relative three-body Schrödinger equation $H_{\text {rel }} \Psi=E_{3 \mathrm{~b}} \Psi$, where

$$
H_{\mathrm{rel}}=H_{\mathrm{rel}, 0}+V_{\mathrm{int}}
$$

with

$$
H_{\mathrm{rel}, 0}=H_{0}(\mathbf{r}, \mu)+H_{0}(\mathbf{R}, \mu)
$$

In Eq. (6), $\mu$ is the two-body reduced mass, $\mu=\mathcal{M} / 2$, and the relative Jacobi coordinates $\mathbf{r}$ and $\mathbf{R}$ are defined through $\mathbf{r}=\mathbf{r}_{31}$ and $\mathbf{R}=\frac{2}{\sqrt{3}}\left(\frac{\mathbf{r}_{1}+\mathbf{r}_{3}}{2}-\mathbf{r}_{2}\right)$. Depending on the context, we use either $\mathbf{r}$ and $\mathbf{R}$ or $\mathbf{r}_{31}$ and $\mathbf{r}_{32}$ to describe the relative degrees of freedom of the three-body system. 
To determine the relative three-body wave function $\Psi(\mathbf{r}, \mathbf{R})$, we take advantage of the fact that the solutions to the "unperturbed" relative Hamiltonian $H_{\text {rel,0 }}$ are known and consider the Lippmann-Schwinger equation (see, e.g., Ref. [8])

$$
\Psi(\mathbf{r}, \mathbf{R})=-\int G\left(E_{3 \mathrm{~b}} ; \mathbf{r}, \mathbf{R} ; \mathbf{r}^{\prime}, \mathbf{R}^{\prime}\right) V_{\mathrm{int}}\left(\mathbf{r}^{\prime}, \mathbf{R}^{\prime}\right) \Psi\left(\mathbf{r}^{\prime}, \mathbf{R}^{\prime}\right) d \mathbf{r}^{\prime} d \mathbf{R}^{\prime}
$$

The Green's function $G$ for the two "pseudoparticles" of mass $\mu$ associated with the Jacobi vectors $\mathbf{r}$ and $\mathbf{R}$ is defined in terms of the eigenstates $\Phi_{\boldsymbol{\lambda}_{1}}(\mathbf{r}) \Phi_{\boldsymbol{\lambda}_{2}}(\mathbf{R})$ and the eigenenergies $E_{\boldsymbol{\lambda}_{1}}+E_{\boldsymbol{\lambda}_{2}}$ of $H_{\text {rel, } 0}$,

$$
G\left(E_{3 \mathrm{~b}} ; \mathbf{r}, \mathbf{R} ; \mathbf{r}^{\prime}, \mathbf{R}^{\prime}\right)=\sum_{\boldsymbol{\lambda}_{1}, \boldsymbol{\lambda}_{2}} \frac{\Phi_{\boldsymbol{\lambda}_{1}}^{*}\left(\mathbf{r}^{\prime}\right) \Phi_{\boldsymbol{\lambda}_{2}}^{*}\left(\mathbf{R}^{\prime}\right) \Phi_{\boldsymbol{\lambda}_{1}}(\mathbf{r}) \Phi_{\boldsymbol{\lambda}_{2}}(\mathbf{R})}{\left(E_{\boldsymbol{\lambda}_{1}}+E_{\boldsymbol{\lambda}_{2}}\right)-E_{3 \mathrm{~b}}} .
$$

Here, $\boldsymbol{\lambda}$ collectively denotes the quantum numbers needed to label the single-particle harmonic osillator states. In cylindrical coordinates, we have $\boldsymbol{\lambda}=\left(n_{z}, n_{\rho}, m\right)$ with $n_{z}=0,1,2, \cdots, n_{\rho}=0,1,2, \cdots$, and $m=0, \pm 1, \pm 2, \cdots$. The single-particle harmonic oscillator eigenenergies and eigenstates read

$$
E_{\boldsymbol{\lambda}}=\left(n_{z}+\frac{1}{2}\right) \hbar \omega_{z}+\left(2 n_{\rho}+|m|+1\right) \eta \hbar \omega_{z}
$$

and

$$
\Phi_{\boldsymbol{\lambda}}(\mathbf{r})=\varphi_{n_{z}}(z) R_{n_{\rho}, m}(\rho) \frac{e^{i m \phi}}{\sqrt{2 \pi}}
$$

where

$$
\varphi_{n_{z}}(z)=\sqrt{\frac{1}{a_{z} \sqrt{\pi} 2^{n_{z}} n_{z} !}} \exp \left(-\frac{z^{2}}{2 a_{z}^{2}}\right) H_{n_{z}}\left(z / a_{z}\right)
$$

and

$$
R_{n_{\rho}, m}(\rho)=\sqrt{\frac{2 \eta n_{\rho} !}{a_{z}^{2}\left(n_{\rho}+|m|\right) !}} \exp \left(-\frac{\eta \rho^{2}}{2 a_{z}^{2}}\right)\left(\frac{\eta^{1 / 2} \rho}{a_{z}}\right)^{|m|} L_{n_{\rho}}^{(|m|)}\left(\eta \rho^{2} / a_{z}^{2}\right)
$$

In the last two equations, $H_{n_{z}}\left(z / a_{z}\right)$ and $L_{n_{\rho}}^{(|m|)}\left(\eta \rho^{2} / a_{z}^{2}\right)$ denote Hermite and associated Laguerre polynomials, respectively. Throughout most of Secs. II] IV] we use the oscillator energy $E_{z}$ and oscillator length $a_{z}\left[E_{z}=\hbar \omega_{z}\right.$ and $\left.a_{z}=\sqrt{\hbar /\left(\mu \omega_{z}\right)}\right]$ as our energy and length units.

In Eqs. (17)-(12), we employ cylindrical coordinates since this choice allows us to write the Green's function $G$ compactly. However, the two-body $s$-wave interaction potential is most conveniently expressed in spherical coordinates [see Eq. (4)]. Since the pseudopotential $V_{\mathrm{ps}}^{3 \mathrm{D}}(\mathbf{r})$ acts only at a single point, namely at $r=0$, it imposes a boundary condition on the relative three-body wave function $\Psi(\mathbf{r}, \mathbf{R})$ (see, e.g., Ref. [34]),

$$
\left.\Psi(\mathbf{r}, \mathbf{R})\right|_{r \rightarrow 0} \approx \frac{f(\mathbf{R})}{4 \pi a_{z}^{3 / 2}}\left(\frac{a_{z}}{r}-\frac{a_{z}}{a^{3 \mathrm{D}}}\right) .
$$

The unknown function $f(\mathbf{R})$ can be interpreted as the relative wave function of the center of mass of the interacting pair and the third particle. Similarly, the pseudopotential $V_{\mathrm{ps}}^{3 \mathrm{D}}\left(\mathbf{r}_{32}\right)$ imposes a boundary condition on the wave function $\Psi(\mathbf{r}, \mathbf{R})$ when $r_{32} \rightarrow 0$. Since the wave function $\Psi(\mathbf{r}, \mathbf{R})$ must be anti-symmetric under the exchange of the two identical fermions, i.e., $P_{12} \Psi(\mathbf{r}, \mathbf{R})=-\Psi(\mathbf{r}, \mathbf{R})$, where $P_{12}$ exchanges particles 1 and 2 , the properly antisymmetrized boundary condition corresponding to $V_{\mathrm{ps}}^{3 \mathrm{D}}\left(\mathbf{r}_{32}\right)$ reads

$$
\left.\Psi\left(\mathbf{r}_{32}, \mathbf{R}_{32}\right)\right|_{r_{32} \rightarrow 0} \approx-\frac{f\left(\mathbf{R}_{32}\right)}{4 \pi a_{z}^{3 / 2}}\left(\frac{a_{z}}{r_{32}}-\frac{a_{z}}{a^{3 \mathrm{D}}}\right) .
$$

Here, we defined $\mathbf{R}_{32}=\frac{2}{\sqrt{3}}\left(\frac{\mathbf{r}_{2}+\mathbf{r}_{3}}{2}-\mathbf{r}_{1}\right)$.

To simplify the right hand side of Eq. (7), we impose the limiting behaviors of $\Psi\left(\mathbf{r}^{\prime}, \mathbf{R}^{\prime}\right)$ for $r_{31}^{\prime} \rightarrow 0$ and $r_{32}^{\prime} \rightarrow 0$, and expand $f\left(\mathbf{R}^{\prime}\right)$ in terms of the non-interacting harmonic oscillator functions, $f\left(\mathbf{R}^{\prime}\right)=\sum_{\boldsymbol{\lambda}^{\prime}} f_{\boldsymbol{\lambda}^{\prime}} \Phi_{\boldsymbol{\lambda}^{\prime}}\left(\mathbf{R}^{\prime}\right)$. Using Eq. (8) for $G$ and orthonormality of the single-particle harmonic oscillator functions, we find

$$
\Psi(\mathbf{r}, \mathbf{R})=\frac{E_{z} a_{z}^{3 / 2}}{2} \sum_{\boldsymbol{\lambda}} f_{\boldsymbol{\lambda}}\left[\mathcal{G}^{3 \mathrm{D}}\left(E_{3 \mathrm{~b}}-E_{\boldsymbol{\lambda}} ; \mathbf{r} ; \mathbf{0}\right) \Phi_{\boldsymbol{\lambda}}(\mathbf{R})-\mathcal{G}^{3 \mathrm{D}}\left(E_{3 \mathrm{~b}}-E_{\boldsymbol{\lambda}} ; \frac{\mathbf{r}+\sqrt{3} \mathbf{R}}{2} ; \mathbf{0}\right) \Phi_{\boldsymbol{\lambda}}\left(\frac{\sqrt{3} \mathbf{r}-\mathbf{R}}{2}\right)\right]
$$


Here, we used that $\mathbf{r}_{32}$ can be written as $(\mathbf{r}+\sqrt{3} \mathbf{R}) / 2$ and introduced the one-body Green's function $\mathcal{G}^{3 \mathrm{D}}\left(E ; \mathbf{r} ; \mathbf{r}^{\prime}\right)$ for the pseudoparticle of mass $\mu$ that is associated with the relative distance vector $\mathbf{r}^{\prime}$,

$$
\mathcal{G}^{3 \mathrm{D}}\left(E ; \mathbf{r} ; \mathbf{r}^{\prime}\right)=\sum_{\boldsymbol{\lambda}^{\prime}} \frac{\Phi_{\boldsymbol{\lambda}^{\prime}}^{*}\left(\mathbf{r}^{\prime}\right) \Phi_{\boldsymbol{\lambda}^{\prime}}(\mathbf{r})}{E_{\boldsymbol{\lambda}^{\prime}}-E} .
$$

The one-body Green's function $\mathcal{G}^{3 \mathrm{D}}\left(E_{2 \mathrm{~b}} ; \mathbf{r} ; \mathbf{r}^{\prime}\right)$ with $\mathbf{r}^{\prime}=\mathbf{0}$ coincides with the solution to the relative Schrödinger equation for two particles under harmonic confinement interacting through the zero-range pseudopotential $V_{\mathrm{ps}}^{3 \mathrm{D}}(\mathbf{r})$ with $s$-wave scattering length $a^{3 \mathrm{D}}$ and relative two-body energy $E_{2 \mathrm{~b}} \cdot \mathcal{G}^{3 \mathrm{D}}(E ; \mathbf{r} ; \mathbf{0})$ is known for all aspect ratios $\eta[6,7]$ (see also Secs. III and IV).

To determine the expansion coefficients $f_{\boldsymbol{\lambda}}$, we apply the operation $\left.\frac{\partial}{\partial r}(r \cdot)\right|_{r \rightarrow 0}$ to the left hand side and the right hand side of Eq. (15), i.e., we multiply both sides of Eq. (15) by $r$, then apply the derivative operator and lastly take the limit $r \rightarrow 0$. Defining

$$
\mathcal{F}^{3 \mathrm{D}}\left(\epsilon_{\boldsymbol{\lambda}}, \eta\right)=\left.2 \pi E_{z} a_{z}^{3} \frac{\partial}{\partial r}\left\{r \mathcal{G}^{3 \mathrm{D}}\left(\left[\epsilon_{\boldsymbol{\lambda}}+\eta+1 / 2\right] E_{z} ; \mathbf{r} ; \mathbf{0}\right)\right\}\right|_{r \rightarrow 0}
$$

with $\left(\epsilon_{\boldsymbol{\lambda}}+\eta+1 / 2\right) E_{z}=E_{3 \mathrm{~b}}-E_{\boldsymbol{\lambda}}$, we find

$$
\begin{aligned}
& -\frac{a_{z}}{2 \pi a^{3 \mathrm{D}}} \sum_{\boldsymbol{\lambda}^{\prime}} f_{\boldsymbol{\lambda}^{\prime}} \Phi_{\boldsymbol{\lambda}^{\prime}}(\mathbf{R})= \\
& \sum_{\boldsymbol{\lambda}^{\prime}} f_{\boldsymbol{\lambda}^{\prime}}\left\{\frac{1}{2 \pi} \mathcal{F}^{3 \mathrm{D}}\left(\epsilon_{\boldsymbol{\lambda}^{\prime}}, \eta\right) \Phi_{\boldsymbol{\lambda}^{\prime}}(\mathbf{R})-E_{z} a_{z}^{3} \mathcal{G}^{3 \mathrm{D}}\left(\left[\epsilon_{\boldsymbol{\lambda}^{\prime}}+\eta+1 / 2\right] E_{z} ; \frac{\sqrt{3}}{2} \mathbf{R} ; \mathbf{0}\right) \Phi_{\boldsymbol{\lambda}^{\prime}}\left(\frac{-\mathbf{R}}{2}\right)\right\} .
\end{aligned}
$$

The quantity $\epsilon_{\boldsymbol{\lambda}^{\prime}}$ can be interpreted as a non-integer quantum number associated with the interacting pair. If we multiply Eq. (18) by $\Phi_{\boldsymbol{\lambda}}^{*}(\mathbf{R})$ and integrate over $\mathbf{R}$, we find an implicit eigenequation for the relative three-body energy $E_{3 \mathrm{~b}}$ or equivalently, the non-integer quantum number $\epsilon_{\boldsymbol{\lambda}}$,

$$
\sum_{\boldsymbol{\lambda}^{\prime}}\left[I_{\boldsymbol{\lambda}, \boldsymbol{\lambda}^{\prime}}^{3 \mathrm{D}}\left(\epsilon_{\boldsymbol{\lambda}^{\prime}}\right)-\mathcal{F}^{3 \mathrm{D}}\left(\epsilon_{\boldsymbol{\lambda}}, \eta\right) \delta_{\boldsymbol{\lambda}, \boldsymbol{\lambda}^{\prime}}\right] f_{\boldsymbol{\lambda}^{\prime}}=\frac{a_{z}}{a^{3 \mathrm{D}}} f_{\boldsymbol{\lambda}}
$$

where

$$
I_{\boldsymbol{\lambda}, \boldsymbol{\lambda}^{\prime}}^{3 \mathrm{D}}\left(\epsilon_{\boldsymbol{\lambda}^{\prime}}\right)=2 \pi E_{z} a_{z}^{3} \int \mathcal{G}^{3 \mathrm{D}}\left(\left[\epsilon_{\boldsymbol{\lambda}^{\prime}}+\eta+1 / 2\right] E_{z} ; \frac{\sqrt{3}}{2} \mathbf{R} ; \mathbf{0}\right) \Phi_{\boldsymbol{\lambda}^{\prime}}\left(\frac{-\mathbf{R}}{2}\right) \Phi_{\boldsymbol{\lambda}}^{*}(\mathbf{R}) d \mathbf{R}
$$

and $\delta_{\boldsymbol{\lambda}, \boldsymbol{\lambda}^{\prime}}$ is the Kronecker delta symbol. The determination of $I_{\boldsymbol{\lambda}, \boldsymbol{\lambda}^{\prime}}^{3 \mathrm{D}}\left(\epsilon_{\boldsymbol{\lambda}^{\prime}}\right)$ and $\mathcal{F}^{3 \mathrm{D}}\left(\epsilon_{\boldsymbol{\lambda}^{\prime}}, \eta\right)$ for $\eta>1$ and $\eta<1$ is discussed in Secs. III and IV respectively.

Equation (19) can be interpreted as a matrix equation with eigenvalues $a_{z} / a^{3 \mathrm{D}}$ and eigenvectors $f_{\boldsymbol{\lambda}}[\underline{8}$, [14]. In practice, we first calculate the matrix elements $I_{\boldsymbol{\lambda}, \lambda^{\prime}}^{3 \mathrm{D}}\left(\epsilon_{\boldsymbol{\lambda}^{\prime}}\right)$ in Eq. (19) for a given three-body energy $E_{3 \mathrm{~b}}$ and obtain the corresponding scattering lengths for this energy by diagonalizing the matrix with elements $I_{\boldsymbol{\lambda}, \boldsymbol{\lambda}^{\prime}}^{3 \mathrm{D}}\left(\epsilon_{\boldsymbol{\lambda}^{\prime}}\right)-\mathcal{F}^{3 \mathrm{D}}\left(\epsilon_{\boldsymbol{\lambda}}, \eta\right) \delta_{\boldsymbol{\lambda}, \boldsymbol{\lambda}^{\prime}}$. This step is repeated for several three-body energies. Lastly, we invert $a^{3 \mathrm{D}}\left(E_{3 \mathrm{~b}}\right)$ to get $E_{3 \mathrm{~b}}\left(a^{3 \mathrm{D}}\right)$, i.e., to get the threebody energies as a function of the $s$-wave scattering length.

Equation (19) has a simple physical interpretation. If the interaction between particles 2 and 3 is turned off, the matrix $I_{\boldsymbol{\lambda}, \boldsymbol{\lambda}^{\prime}}^{3 \mathrm{D}}\left(\epsilon_{\boldsymbol{\lambda}^{\prime}}\right)$ vanishes and the solution reduces to that of an interacting pair (particles 1 and 3) and a noninteracting spectator particle (particle 2). The relative energy $\left(\epsilon_{\boldsymbol{\lambda}^{\prime}}+\eta+1 / 2\right) E_{z}$ of the pair is determined by solving the relative two-body eigenequation $\mathcal{F}^{3 \mathrm{D}}\left(\epsilon_{\boldsymbol{\lambda}^{\prime}}, \eta\right)=-a_{z} / a^{3 \mathrm{D}}$. The matrix $I_{\boldsymbol{\lambda}, \boldsymbol{\lambda}^{\prime}}^{3 \mathrm{D}}\left(\epsilon_{\boldsymbol{\lambda}^{\prime}}\right)$ thus arises from the fact that particle 3 not only interacts with particle 1 but also with particle 2. Correspondingly, the terms in Eq. (19) that contain $I_{\boldsymbol{\lambda}, \boldsymbol{\lambda}^{\prime}}^{3 \mathrm{D}}\left(\epsilon_{\boldsymbol{\lambda}^{\prime}}\right)$ can be interpreted as exchange terms that arise from exchanging particles 1 and 2 [14].

For $\eta=1$, the function $\mathcal{F}^{3 \mathrm{D}}\left(\epsilon_{\boldsymbol{\lambda}}, \eta\right)$ is given in Table $\llbracket$ and the evaluation of $I_{\boldsymbol{\lambda}, \boldsymbol{\lambda}^{\prime}}^{3 \mathrm{D}}\left(\epsilon_{\boldsymbol{\lambda}^{\prime}}\right)$ has been discussed in detail in Ref. [15]. The $\eta \neq 1$ cases are discussed in Secs. III] and [IV] For a spherically symmetic system with $\eta=1$, the total relative angular momentum quantum number $L$, the corresponding projection quantum number $M$ and the parity $\Pi$ are good quantum numbers, and the eigenvalue equation can be solved for each $L$ and $M$ combination separately using spherical coordinates [8]. For a fixed $L$ and $M, \boldsymbol{\lambda}=(n, l, m)$ and $\boldsymbol{\lambda}^{\prime}=\left(n^{\prime}, l^{\prime}, m^{\prime}\right)$ in Eq. (19) are constrained by $l=l^{\prime}=L$ and $m=m^{\prime}=M$. The parity of the three-body system is given by $\Pi=(-1)^{L}$. 
TABLE I: Two-body properties in three dimensions ( $s$-wave channel), two dimensions $(m=0$ channel), and one dimension (even parity channel). $\psi(\mathbf{q})$ denotes the relative two-body wave function and $\mu$ the two-body reduced mass. q stands for $\mathbf{r}, \boldsymbol{\rho}$, and $z$ in three, two, and one dimensions, respectively. For 3D, 2D and 1D, we have $\epsilon=E_{2 \mathrm{~b}} / E_{z}-\eta-1 / 2, \epsilon=E_{2 \mathrm{~b}} / E_{\rho}-1$ and $\epsilon=E_{2 \mathrm{~b}} / E_{z}-1 / 2$, respectively, where $E_{2 \mathrm{~b}}$ denotes the relative two-body energy. $\psi_{g}$ denotes the digamma function and $\gamma$ the Euler constant, $\gamma \approx 0.577$.

\begin{tabular}{llll}
\hline \hline & $3 \mathrm{D}$ & $2 \mathrm{D}$ & $1 \mathrm{D}$ \\
\hline$V_{\mathrm{ps}}$ & $g^{3 \mathrm{D}} \delta^{(3)}(\mathbf{r}) \frac{\partial}{\partial r} r$ & $g^{2 \mathrm{D}} \delta^{(2)}(\boldsymbol{\rho}) \frac{\partial}{\partial \rho} \rho$ & $g^{1 \mathrm{D}} \delta(z)$ \\
\hline$g$ & $2 \pi \frac{\hbar^{2}}{\mu} a^{3 \mathrm{D}}$ & $\pi \frac{\hbar^{2}}{\mu}\left[\ln \left(\frac{\rho}{a^{2 \mathrm{D}}}\right)+1\right]^{-1}$ & $-\frac{\hbar^{2}}{\mu} \frac{1}{a^{1 \mathrm{D}}}$ \\
\hline$\left.\psi(\mathbf{q})\right|_{|\mathbf{q}| \rightarrow 0}$ & $\propto\left(\frac{1}{r}-\frac{1}{a^{3 \mathrm{D}}}\right)$ & $\propto\left[\ln \left(a^{2 \mathrm{D}}\right)-\ln (\rho)\right]$ & $\propto\left(z-a^{1 \mathrm{D}}\right)$ \\
\hline Bethe-Peierls B.C. & $\left.\frac{\partial(r \psi)}{\partial r}\right|_{r \rightarrow 0}=\left.\frac{-1}{a^{3 \mathrm{D}}}(r \psi)\right|_{r \rightarrow 0}$ & $\left.\rho \frac{\partial \psi}{\partial \rho}\right|_{\rho \rightarrow 0}=\left.\frac{\psi}{\ln \left(\rho / a^{2 \mathrm{D}}\right)}\right|_{\rho \rightarrow 0}$ & $\left.\frac{d \psi}{d z}\right|_{z \rightarrow 0}=\left.\frac{-1}{a^{1 \mathrm{D}}} \psi\right|_{z \rightarrow 0}$ \\
\hline $\mathcal{F}$ & $\mathcal{F}^{3 \mathrm{D}}(\epsilon, \eta)=2 \pi E_{z} a_{z}^{3}$ & $\mathcal{F}^{2 \mathrm{D}}(\epsilon)=\pi E_{\rho} a_{\rho}^{2}$ & $\mathcal{F}^{1 \mathrm{D}}(\epsilon)=E_{z} a_{z}$ \\
& $\left\{\frac{\partial}{\partial r}\left[r \mathcal{G}^{3 \mathrm{D}}\left(\left[\epsilon+\eta+\frac{1}{2}\right] E_{z} ; \mathbf{r} ; \mathbf{0}\right)\right]\right\}_{r \rightarrow 0}$ & $\left\{\mathcal{G}^{2 \mathrm{D}}\left([\epsilon+1] E_{\rho} ; \rho ; 0\right)+\ln \left(\rho / a_{\rho}\right)\right\}_{\rho \rightarrow 0}$ & $\left\{\mathcal{G}^{\mathrm{D} \mathrm{D}}\left(\left[\epsilon+\frac{1}{2}\right] E_{z} ; z ; 0\right)\right\}_{z \rightarrow 0}$ \\
\hline & $\mathcal{F}^{3 \mathrm{D}}(\epsilon, 1)=\frac{-2 \Gamma(-\epsilon)}{\Gamma(-\epsilon / 2-1 / 2)}$ & $\mathcal{F}^{2 \mathrm{D}}(\epsilon)=-\frac{1}{2} \psi_{g}(-\epsilon / 2)-\gamma$ & $\mathcal{F}^{1 \mathrm{D}}(\epsilon)=\frac{\Gamma(-\epsilon / 2)}{2 \Gamma(-\epsilon / 2+1 / 2)}$ \\
\hline two-body energy & $\mathcal{F}^{3 \mathrm{D}}(\epsilon, \eta)=-a_{z} / a^{3 \mathrm{D}}$ & $\mathcal{F}^{2 \mathrm{D}}(\epsilon)=\ln \left(a^{2 \mathrm{D}} / a_{\rho}\right)$ & $\mathcal{F}^{1 \mathrm{D}}(\epsilon)=a^{1 \mathrm{D}} / a_{z}$ \\
\hline \hline
\end{tabular}

We emphasize that the outlined formalism makes no approximations, i.e., Eq. (19) with $I_{\boldsymbol{\lambda}, \boldsymbol{\lambda}^{\prime}}^{3 \mathrm{D}}\left(\epsilon_{\boldsymbol{\lambda}^{\prime}}\right)$ given by Eq. (20) describes all eigenstates of $H_{\text {rel }}$ [see Eq. (5)] that are affected by the interactions. In particular, all "channel couplings" are accounted for. In practice, the construction of the matrix $I_{\boldsymbol{\lambda}, \boldsymbol{\lambda}^{\prime}}^{3 \mathrm{D}}\left(\epsilon_{\boldsymbol{\lambda}^{\prime}}\right)$ requires one to choose a maximum for $\boldsymbol{\lambda}$ and $\lambda^{\prime}$, or alternatively, a cutoff for the single-particle energy $E_{\boldsymbol{\lambda}}$. As has been shown in Ref. [15], good convergence is achieved for a relatively small number of "basis functions" for $\eta=1$. As we show below, good convergence is also obtained for anisotropic confinement geometries.

The formalism outlined can also be applied to strictly one-dimensional and strictly two-dimensional systems. Table \defines the one-dimensional and two-dimensional pseudopotentials as well as a number of key properties of the corresponding relative two-body system. Making the appropriate changes in the outlined derivation and using the properties listed in Table 【. we find for strictly one-dimensional systems

$$
\sum_{n_{z}^{\prime}=0}^{\infty}\left[I_{n_{z}, n_{z}^{\prime}}^{1 \mathrm{D}}\left(\epsilon_{n_{z}^{\prime}}\right)-\mathcal{F}^{1 \mathrm{D}}\left(\epsilon_{n_{z}}\right) \delta_{n_{z}, n_{z}^{\prime}}\right] f_{n_{z}^{\prime}}=-\frac{a^{1 \mathrm{D}}}{a_{z}} f_{n_{z}},
$$

where $\mathcal{F}^{1 \mathrm{D}}$ is defined in Table 【

$$
I_{n_{z}, n_{z}^{\prime}}^{1 \mathrm{D}}\left(\epsilon_{n_{z}^{\prime}}\right)=E_{z} a_{z} \int_{-\infty}^{\infty} \mathcal{G}^{1 \mathrm{D}}\left(\left[\epsilon_{n_{z}^{\prime}}+1 / 2\right] E_{z} ; \frac{\sqrt{3}}{2} z ; 0\right) \varphi_{n_{z}^{\prime}}\left(-\frac{z}{2}\right) \varphi_{n_{z}}^{*}(z) d z,
$$

and $E_{3 \mathrm{~b}}-E_{n_{z}^{\prime}}=\left(\epsilon_{n_{z}^{\prime}}+1 / 2\right) E_{z}$. Here, $E_{n_{z}}$ denotes the single-particle energy of the one-dimensional system, $E_{n_{z}}=$ $\left(n_{z}+1 / 2\right) E_{z}$, and $\mathcal{G}^{1 \mathrm{D}}\left(E ; z ; z^{\prime}\right)$ the one-dimensional even parity single-particle Green's function,

$$
\mathcal{G}^{1 \mathrm{D}}\left(E ; z ; z^{\prime}\right)=\sum_{n_{z}^{\prime}=0}^{\infty} \frac{\varphi_{2 n_{z}^{\prime}}^{*}\left(z^{\prime}\right) \varphi_{2 n_{z}^{\prime}}(z)}{E_{2 n_{z}^{\prime}}-E}
$$

For $z^{\prime}=0$, the single-particle Green's function is given by

$$
\mathcal{G}^{1 \mathrm{D}}(E ; z ; 0)=\frac{1}{2 \sqrt{\pi} E_{z} a_{z}} \exp \left(-\frac{z^{2}}{2 a_{z}^{2}}\right) \Gamma\left(-\frac{E / E_{z}-1 / 2}{2}\right) U\left(-\frac{E / E_{z}-1 / 2}{2}, \frac{1}{2}, \frac{z^{2}}{a_{z}^{2}}\right),
$$

where $\Gamma(x)$ is the Gamma function and $U(a, b, z)$ the confluent hypergeometric function. The strictly one-dimensional relative three-body wave function $\Psi$ is characterized by the parity $\Pi_{z}$. For even parity states, i.e., for states with $\Pi_{z}=1, n_{z}$ and $n_{z}^{\prime}$ in Eq. (21) have to be even. For odd parity states, i.e., for states with $\Pi_{z}=-1, n_{z}$ and $n_{z}^{\prime}$ have to be odd.

Similarly, for strictly two-dimensional systems, expressed in units of $E_{\rho}$ and $a_{\rho}\left[E_{\rho}=\hbar \omega_{\rho}\right.$ and $\left.a_{\rho}=\sqrt{\hbar /\left(\mu \omega_{\rho}\right)}\right]$, we find, in agreement with Ref. [16],

$$
\sum_{n_{\rho}^{\prime}=0}^{\infty}\left[I_{n_{\rho}, n_{\rho}^{\prime}, m}^{2 \mathrm{D}}\left(\epsilon_{n_{\rho}^{\prime}, m}\right)-\mathcal{F}^{2 \mathrm{D}}\left(\epsilon_{n_{\rho}, m}\right) \delta_{n_{\rho}, n_{\rho}^{\prime}}\right] f_{n_{\rho}^{\prime}, m}=\ln \left(\frac{a_{\rho}}{a^{2 \mathrm{D}}}\right) f_{n_{\rho}, m},
$$


where $\mathcal{F}^{2 \mathrm{D}}$ is defined in Table $\llbracket$

$$
I_{n_{\rho}, n_{\rho}^{\prime}, m}^{2 \mathrm{D}}\left(\epsilon_{n_{\rho}^{\prime}, m}\right)=(-1)^{m} \pi E_{\rho} a_{\rho}^{2} \int_{0}^{\infty} \mathcal{G}^{2 \mathrm{D}}\left(\left[\epsilon_{n_{\rho}^{\prime}, m}+1\right] E_{\rho} ; \frac{\sqrt{3}}{2} \rho ; 0\right) R_{n_{\rho}^{\prime}, m}\left(\frac{\rho}{2}\right) R_{n_{\rho}, m}(\rho) \rho d \rho,
$$

and $E_{3 \mathrm{~b}}-E_{n_{\rho}^{\prime}, m}=\left(\epsilon_{n_{\rho}^{\prime}, m}+1\right) E_{\rho}$. Here, $E_{n_{\rho}, m}$ denotes the single-particle energy of the two-dimensional system, $E_{n_{\rho}, m}=\left(2 n_{\rho}+|m|+1\right) E_{\rho}$. The two-dimensional single-particle Green's function $\mathcal{G}^{2 \mathrm{D}}\left(E ; \rho ; \rho^{\prime}\right)$ is defined analogously to the three- and one-dimensional counterparts [see Eqs. (16) and (23)]. For $\rho^{\prime}=0$ and states affected by the zero-range $s$-wave interactions [6, 7], one finds

$$
\mathcal{G}^{2 \mathrm{D}}(E ; \rho ; 0)=\frac{1}{2 \pi E_{\rho} a_{\rho}^{2}} \exp \left(-\frac{\rho^{2}}{2 a_{\rho}^{2}}\right) \Gamma\left(-\frac{E / E_{\rho}-1}{2}\right) U\left(-\frac{E / E_{\rho}-1}{2}, 1, \frac{\rho^{2}}{a_{\rho}^{2}}\right) .
$$

The strictly two-dimensional relative three-body wave function is characterized by the projection quantum number $M$ and the parity $\Pi_{\rho}, \Pi_{\rho}=(-1)^{M}$. For a fixed $M, m$ in Eq. (25) is constrained to the value $m=M$. The next two sections analyze, utilizing our results for strictly one- and two-dimensional systems, Eq. (19) for cigar- and pancake-shaped traps.

\section{CIGAR-SHAPED TRAP}

To apply the formalism reviewed in Sec. II to axially symmetric traps, we need the explicit forms of the functions $\mathcal{G}^{3 \mathrm{D}}\left(\left[\epsilon_{\boldsymbol{\lambda}}+\eta+1 / 2\right] E_{z} ; \mathbf{r} ; \mathbf{0}\right)$ and $\mathcal{F}^{3 \mathrm{D}}\left(\epsilon_{\boldsymbol{\lambda}}, \eta\right)$, that is, the relative solutions to the trapped two-body system. For cigarshaped traps $(\eta>1)$, it is convenient to write $\mathcal{G}^{3 \mathrm{D}}$ as $[6,7]$

$$
\mathcal{G}^{3 \mathrm{D}}\left(\left[\epsilon_{\boldsymbol{\lambda}}+\eta+1 / 2\right] E_{z} ; \mathbf{r} ; \mathbf{0}\right)=\frac{\eta}{\pi a_{z}^{2}} \exp \left(-\frac{\eta \rho^{2}}{2 a_{z}^{2}}\right) \sum_{j=0}^{\infty} L_{j}\left(\eta \rho^{2} / a_{z}^{2}\right) \mathcal{G}^{1 \mathrm{D}}\left(\left[\epsilon_{\boldsymbol{\lambda}}-2 \eta j+1 / 2\right] E_{z} ; z ; 0\right),
$$

where $\mathcal{G}^{1 \mathrm{D}}(E ; z ; 0)$ is defined in Eq. (24). Using Eq. (28) in Eq. (20), we obtain

$$
\begin{array}{r}
I_{\boldsymbol{\lambda}, \boldsymbol{\lambda}^{\prime}}^{3 \mathrm{D}}\left(\epsilon_{\boldsymbol{\lambda}^{\prime}}\right)=\sqrt{2 \eta}(-1)^{m} \delta_{m, m^{\prime}} \times \\
\lim _{j_{\max } \rightarrow \infty} \sum_{j=0}^{j_{\max }} I_{n_{z}, n_{z}^{\prime}}^{\mathrm{c}}\left(\epsilon_{\boldsymbol{\lambda}^{\prime}}, j\right) I_{n_{\rho}, n_{\rho}^{\prime}, m}^{\mathrm{c}}(j),
\end{array}
$$

where

$$
I_{n_{z}, n_{z}^{\prime}}^{\mathrm{c}}\left(\epsilon_{\boldsymbol{\lambda}^{\prime}}, j\right)=E_{z} a_{z} \int_{-\infty}^{\infty} \mathcal{G}^{1 \mathrm{D}}\left(\left[\epsilon_{\boldsymbol{\lambda}^{\prime}}-2 \eta j+1 / 2\right] E_{z} ; \frac{\sqrt{3}}{2} z ; 0\right) \varphi_{n_{z}^{\prime}}\left(-\frac{z}{2}\right) \varphi_{n_{z}}(z) d z
$$

and

$$
\begin{aligned}
& I_{n_{\rho}, n_{\rho}^{\prime}, m}^{\mathrm{c}}(j)= \\
& a_{z} \int_{0}^{\infty} R_{j, 0}\left(\frac{\sqrt{3}}{2} \rho\right) R_{n_{\rho}^{\prime}, m}\left(\frac{\rho}{2}\right) R_{n_{\rho}, m}(\rho) \rho d \rho .
\end{aligned}
$$

The evaluation of the integrals $I_{n_{z}, n_{z}^{\prime}}^{\mathrm{c}}\left(\epsilon_{\boldsymbol{\lambda}^{\prime}}, j\right)$ and $I_{n_{\rho}, n_{\rho}^{\prime}, m}^{\mathrm{c}}(j)$ is discussed in Appendix $\mathrm{A}$ The superscript "c" indicates that the integrals apply to cigar-shaped systems; for pancake-shaped systems (see Sec. IV]), we introduce the integrals $I_{n_{\rho}, n_{\rho}^{\prime}, m}^{\mathrm{p}}\left(\epsilon_{\boldsymbol{\lambda}^{\prime}}, j\right)$ and $I_{n_{z}, n_{z}^{\prime}}^{\mathrm{p}}(j)$ instead.

Although it is possible to calculate $\mathcal{F}^{3 \mathrm{D}}\left(\epsilon_{\boldsymbol{\lambda}}, \eta\right)$ numerically for any trap aspect ratio $\eta$, we restrict ourselves to integer aspect ratios for simplicity. For traps with integer aspect ratio, an exact analytical expression for $\mathcal{F}^{3 \mathrm{D}}\left(\epsilon_{\boldsymbol{\lambda}}, \eta\right)$ is known [6, 7],

$$
\begin{array}{r}
\mathcal{F}^{3 \mathrm{D}}\left(\epsilon_{\boldsymbol{\lambda}}, \eta\right)=-2 \frac{\Gamma\left(-\frac{\epsilon_{\boldsymbol{\lambda}}}{2}\right)}{\Gamma\left(-\frac{1}{2}-\frac{\epsilon_{\boldsymbol{\lambda}}}{2}\right)}+\frac{\Gamma\left(-\frac{\epsilon_{\boldsymbol{\lambda}}}{2}\right)}{\Gamma\left(\frac{1}{2}-\frac{\epsilon_{\boldsymbol{\lambda}}}{2}\right)} \times \\
\sum_{k=1}^{\eta-1}{ }_{2} F_{1}\left(1,-\frac{\epsilon_{\boldsymbol{\lambda}}}{2} ; \frac{1}{2}-\frac{\epsilon_{\boldsymbol{\lambda}}}{2} ; \exp \left(\frac{2 \pi \imath k}{\eta}\right)\right),
\end{array}
$$




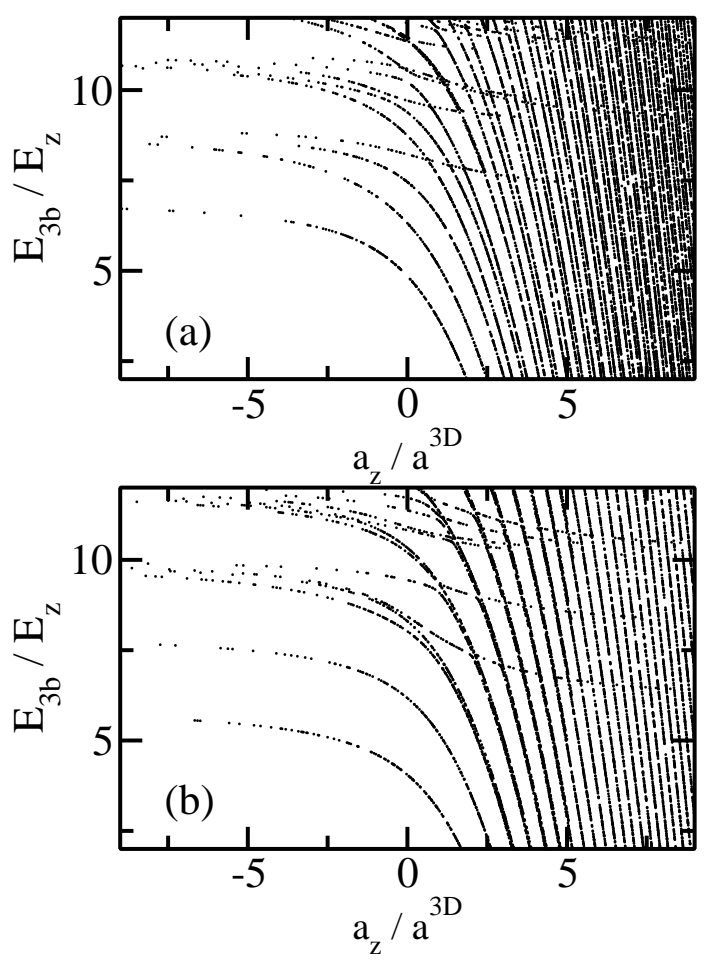

FIG. 1: Relative three-body energies $E_{3 \mathrm{~b}} / E_{z}$ as a function of the inverse scattering length $a_{z} / a^{3 \mathrm{D}}$ for a cigar-shaped trap with aspect ratio $\eta=2$ and (a) $M=0$ and $\Pi_{z}=+1$, and (b) $M=0$ and $\Pi_{z}=-1$.

where ${ }_{2} F_{1}(a, b ; c ; z)$ is the hypergeometric function [47]. Knowing $I_{\boldsymbol{\lambda}, \boldsymbol{\lambda}^{\prime}}^{3 \mathrm{D}}\left(\epsilon_{\boldsymbol{\lambda}^{\prime}}\right)$ and $\mathcal{F}^{3 \mathrm{D}}\left(\epsilon_{\boldsymbol{\lambda}^{\prime}}, \eta\right)$, Eq. [19) can be diagonalized separately for each $\left(\Pi_{z}, M, \Pi_{\rho}\right)$ combination. We recall from Sec. П that $\boldsymbol{\lambda}=\left(n_{z}, n_{\rho}, m\right)$ and $\boldsymbol{\lambda}^{\prime}=\left(n_{z}^{\prime}, n_{\rho}^{\prime}, m^{\prime}\right)$. The $m$ and $m^{\prime}$ values are constrained by $m=m^{\prime}=M$. Moreover, for $\Pi_{z}=+1$ and $\Pi_{z}=-1$, we have $n_{z}=n_{z}^{\prime}=$ even and $n_{z}=n_{z}^{\prime}=o d d$, respectively.

Figure 1 shows the three-body relative energies $E_{3 \mathrm{~b}} / E_{z}$ for $\eta=2$ for states with (a) $M=0$ and $\Pi_{z}=+1$ and (b) $M=0$ and $\Pi_{z}=-1$ as a function of the inverse scattering length $a_{z} / a^{3 \mathrm{D}}$. The non-interacting limit is approached when $\left(a^{3 \mathrm{D}}\right)^{-1} \rightarrow \pm \infty$, and the infinitely strongly-interacting regime for $\left(a^{3 \mathrm{D}}\right)^{-1}=0$ (center of the figure). For each fixed projection quantum number $M$, we include around 840 basis functions. This corresponds to a cutoff of around $(82+2 M) E_{z}$ for the single-particle energy $E_{\boldsymbol{\lambda}}$. We find that $j_{\max } \gtrsim 30$ yields converged values for $I_{\boldsymbol{\lambda}, \boldsymbol{\lambda}^{\prime}}^{3 \mathrm{D}}\left(\epsilon_{\boldsymbol{\lambda}^{\prime}}\right)$, Eq. (29). For small $\left|a^{3 \mathrm{D}} / a_{z}\right|$ ( $a^{3 \mathrm{D}}$ positive and negative), our eigenenergies agree with those obtained within first-order perturbation theory. Our analysis shows that the energy of the ground state at unitarity has a relative error of the order of $10^{-5}$. The accuracy decreases with increasing energy. For example, for energies around $20 E_{z}$, the relative accuracy at unitarity is of the order of $10^{-4}$.

The eigenstates fall into one of two categories: atom-dimer states and atom-atom-atom states. The eigenenergies associated with the former are negative for large positive $a_{z} / a^{3 \mathrm{D}}$ while those associated with the latter remain positive for large positive $a_{z} / a^{3 \mathrm{D}}$. The energy spectra shown in Fig. 1 exhibit sequences of avoided crossings. To resolve these crossings, a fairly fine mesh in the three-body energy is needed. In the $\left(a^{3 \mathrm{D}}\right)^{-1} \rightarrow-\infty$ limit, the lowest $M=0$ state has negative parity in $z$, i.e., $\Pi_{z}=-1$. This is a direct consequence of the fact that the two identical fermions cannot occupy the same single particle state. In the $\left(a^{3 \mathrm{D}}\right)^{-1} \rightarrow+\infty$ limit, in contrast, the lowest $M=0$ state has positive parity in $z$, i.e., $\Pi_{z}=+1$. This is a direct consequence of the fact that the system consists, effectively, of a dimer and an atom.

The main part of Fig. 2 shows the relative energy of the energetically lowest-lying state, the so-called crossover curve, of the three-body system with $M=0$ for various aspect ratios of the trap $(\eta=2, \cdots, 10)$ as a function of the inverse scattering length $a_{z} / a^{3 \mathrm{D}}$. For comparative purposes, we subtract the ground state energy of $2 \eta E_{z}$ of the strictly two-dimensional non-interacting system, that is, the energy that the system would have in the $\rho$-direction if the dynamics in the tight confinement direction were frozen, from the full three-dimensional energy. In Fig. 2, asterisks 


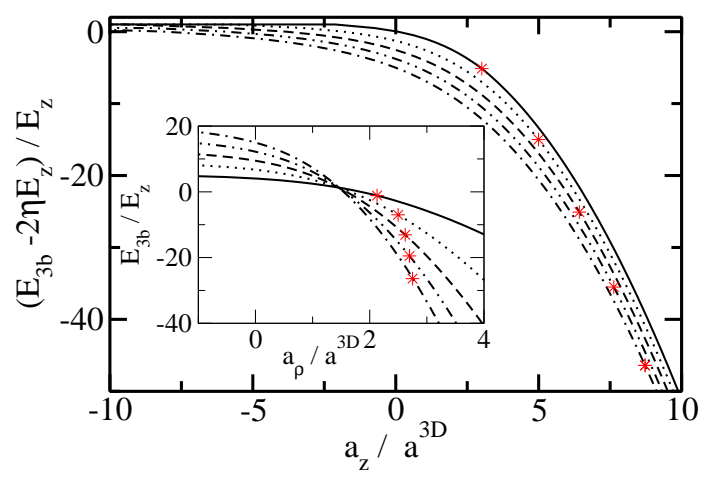

FIG. 2: (Color online) "Crossover curve" of the three-body system with $M=0$, shifted by $2 E_{\rho}=2 \eta E_{z}$, for cigar-shaped traps with $\eta=2$ (solid line), 4 (dotted line), 6 (dashed line), 8 (dash-dot-dotted line), and 10 (dash-dotted line) as a function of $a_{z} / a^{3 \mathrm{D}}$. The scattering lengths at which the parity of the corresponding eigenstate changes from $\Pi_{z}=-1$ ("left side of the graph") to $\Pi_{z}=+1$ ("right side of the graph") are marked by asterisks. At these points, the derivative of the crossover curve is discontinuous; the discontinuities are not visible on the scale shown. The inset shows the (unshifted) crossover curve as a function of $a_{\rho} / a^{3 \mathrm{D}}$.

mark the scattering lengths at which the eigenstate associated with the crossover curve changes from $\Pi_{z}=-1$ to $\Pi_{z}=+1$. With increasing $\eta$, the parity change occurs at larger $a_{z} / a^{3 \mathrm{D}}$ (that is, smaller $a^{3 \mathrm{D}} / a_{z}$ ). The inset of Fig. 2 replots the crossover curves as a function of $a_{\rho} / a^{3 \mathrm{D}}$.

We now discuss the large $\eta$ limit in more detail. Using the limiting behavior of $\mathcal{F}^{3 \mathrm{D}}(\epsilon, \eta)$ for $\eta \gg 1$ and $\eta \gg|\epsilon|[\underline{6}$, 7],

$$
\left.\mathcal{F}^{3 \mathrm{D}}(\epsilon, \eta)\right|_{\eta \gg 1} \approx 2 \eta \mathcal{F}^{1 \mathrm{D}}(\epsilon)+\sqrt{\eta} \zeta(1 / 2),
$$

the two-body eigenequation for the relative energy becomes $[\underline{6}, 7]$

$$
\mathcal{F}^{1 \mathrm{D}}(\epsilon)=\frac{a_{\mathrm{ren}}^{1 \mathrm{D}}}{a_{z}}
$$

where the renormalized one-dimensional scattering length $a_{\text {ren }}^{1 \mathrm{D}}$ is given by [20, 21]

$$
\frac{a_{\mathrm{ren}}^{1 \mathrm{D}}}{a_{z}}=\frac{1}{\sqrt{\eta}}\left[-\frac{a_{\rho}}{2 a^{3 \mathrm{D}}}-\frac{\zeta(1 / 2)}{2}\right] .
$$

Figure 3(a) shows the relative two-body energies for a system with $\eta=10, M=0$ and $\Pi_{z}=+1$ obtained by solving the eigenequation $\mathcal{F}^{3 \mathrm{D}}(\epsilon, \eta=10)=-a_{z} / a^{3 \mathrm{D}}$ [see Eq. (32) for $\mathcal{F}^{3 \mathrm{D}}(\epsilon, \eta)$ ]. Figure 3(b) compares the full three-dimensional energy (solid line) with the energy obtained by solving the strictly one-dimensional eigenequation, Eq. (34), with renormalized one-dimensional scattering length $a_{\mathrm{ren}}^{1 \mathrm{D}}$ (dotted line). To facilitate the comparison, we add the energy of the tight confinement direction to the energy of the one-dimensional system. The agreement is quite good for all scattering lengths. The inset of Fig. 3(b) shows the difference between the strictly one-dimensional energy and the full three-dimensional energy as a function of $a_{z} / a^{3 \mathrm{D}}$. The maximum deviation occurs around unitarity and is of the order of $0.2 \%$.

Next, we discuss the behavior of the three-body system in the large $\eta$ limit. If we use Eqs. (33) and (35) in Eq. (19), we find

$$
\sum_{\boldsymbol{\lambda}^{\prime}}\left[\frac{1}{2 \eta} I_{\boldsymbol{\lambda}, \boldsymbol{\lambda}^{\prime}}^{3 \mathrm{D}}\left(\epsilon_{\boldsymbol{\lambda}^{\prime}}\right)-\mathcal{F}^{1 \mathrm{D}}\left(\epsilon_{\boldsymbol{\lambda}}\right) \delta_{\boldsymbol{\lambda}, \boldsymbol{\lambda}^{\prime}}\right] f_{\boldsymbol{\lambda}^{\prime}}=-\frac{a_{\mathrm{ren}}^{1 \mathrm{D}}}{a_{z}} f_{\boldsymbol{\lambda}}
$$

A straightforward analysis shows that Eq. (36) reduces to its strictly one-dimensional analog if (i) the sum over $\lambda^{\prime}$ is restricted to a sum over $n_{z}^{\prime}\left[\boldsymbol{\lambda}^{\prime}=\left(n_{z}^{\prime}, 0,0\right)\right]$; (ii) the index $\boldsymbol{\lambda}$ is restricted to $\boldsymbol{\lambda}=\left(n_{z}, 0,0\right)$; (iii) the energy $E_{3 \mathrm{~b}}$ is replaced by $E_{3 \mathrm{~b}}-2 \eta E_{z}$; and (iv) $j_{\max }$ in Eq. (29) is set to zero. Under these assumptions, Eq. (36) reduces to Eq. (21) with $a^{1 \mathrm{D}}$ replaced by $a_{\mathrm{ren}}^{1 \mathrm{D}}$. We emphasize that the assumption $\eta \gg\left|\epsilon_{\boldsymbol{\lambda}}\right|$ [see discussion around Eq. (33)] is 

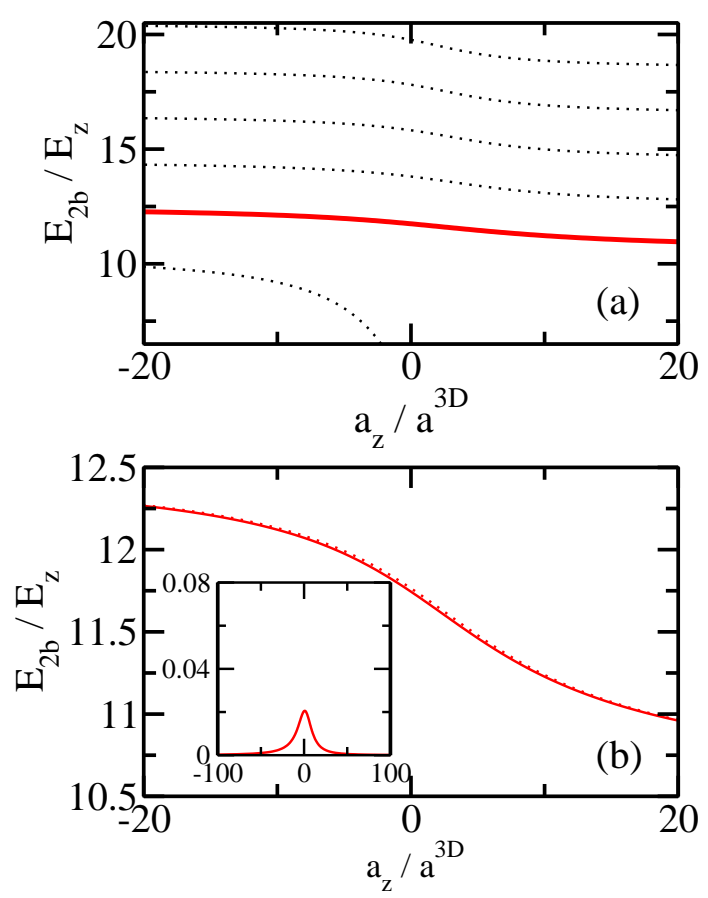

FIG. 3: (Color online) (a) Relative two-body energies $E_{2 \mathrm{~b}} / E_{z}$ as a function of the inverse scattering length $a_{z} / a^{3 \mathrm{D}}$ for a cigarshaped trap with aspect ratio $\eta=10, M=0$ and $\Pi_{z}=+1$. (b) The solid curve from panel (a) is replotted and compared with the energy obtained by solving the strictly one-dimensional eigenequation with renormalized one-dimensional scattering length $a_{\mathrm{ren}}^{1 \mathrm{D}}$ (dotted line; the energy $E_{\rho}=\eta E_{z}$ has been added to allow for a comparison with the full three-dimensional energy). The inset shows the difference between the dotted and solid lines as a function of the inverse scattering length $a_{z} / a^{3 \mathrm{D}}$. The scale of the $y$-axis is identical to that of the inset of Fig. 4(b).

not valid when two atoms form a tight molecule. In this limit, the three-dimensional $s$-wave scattering length, or the size of the dimer, is smaller than the harmonic oscillator length in the transverse direction, which implies that the strictly one-dimensional description is not valid.

Figure 4(a) shows the relative three-body energies for states with $M=0$ and $\Pi_{z}=+1$ as a function of the inverse scattering length $a_{z} / a^{3 \mathrm{D}}$ for a cigar-shaped trap with $\eta=10$. Figure 4 (b) compares the energy of the energetically lowest-lying three-atom state with $\Pi_{z}=+1$ (solid line) [see thick solid line in Fig. 4(a)] with the corresponding state obtained by solving the strictly one-dimensional equation with renormalized one-dimensional scattering length $a_{\mathrm{ren}}^{1 \mathrm{D}}$. We also include the energy of one of the eigenstates with $M=0$ and $\Pi_{z}=-1$ (dashed line). The inset shows the difference between the energy obtained within the strictly one-dimensional and the full three-dimensional frameworks. The maximum of the deviation occurs near unitarity. The agreement between the full three-dimensional and the strictly one-dimensional descriptions is good. Importantly, the deviations for the three-body system with $M=0$ and $\Pi_{z}=+1$ [solid line in the inset of Fig. 4(b)] are only slightly larger than those for the two-body system [inset of Fig. [3(b)], suggesting that the presence of the third atom does not, in a significant manner, reduce the applicability of the strictly one-dimensional framework - at least for states in the low-energy regime characterized as gas-like three-atom states. 


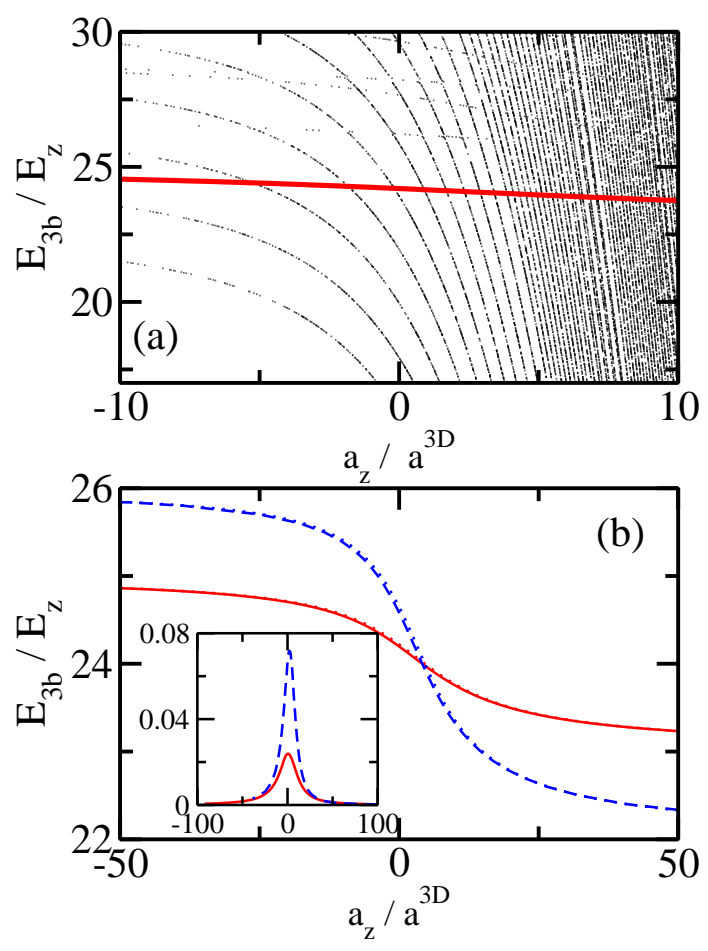

FIG. 4: (Color online) (a) Relative three-body energies $E_{3 \mathrm{~b}} / E_{z}$ as a function of the inverse scattering length $a_{z} / a^{3 \mathrm{D}}$ for a cigar-shaped trap with aspect ratio $\eta=10, M=0$ and $\Pi_{z}=+1$. (b) The solid curve from panel (a) is replotted and compared with the energy obtained by solving the strictly one-dimensional eigenequation with renormalized one-dimensional scattering length $a_{\mathrm{ren}}^{1 \mathrm{D}}$ (the energy of $2 E_{\rho}=2 \eta E_{z}$ has been added to allow for a comparison with the full three-dimensional energy). For comparison, the dashed line shows one of the three-dimensional energy curves for $M=0$ and $\Pi_{z}=-1$ [not shown in panel (a)]; the corresponding strictly one-dimensional energy is shown by a dotted line. The difference between the full three-dimensional and strictly one-dimensional descriptions is hardly visible on the scale shown. Solid and dashed lines in the inset show the differences between the strictly one-dimensional energies [dotted lines in panel (b)] and the full three-dimensional energies [solid and dashed lines in panel (b)] as a function of the inverse scattering length $a_{z} / a^{3 \mathrm{D}}$ for $\Pi_{z}=+1$ and $\Pi_{z}=-1$, respectively.

\section{PANCAKE-SHAPED TRAP}

For pancake-shaped traps with $\eta<1$, we use the following form of the Green's function $\mathcal{G}^{3 \mathrm{D}}$ [6, 7],

$$
\mathcal{G}^{3 \mathrm{D}}\left(\left[\epsilon_{\boldsymbol{\lambda}}+\eta+1 / 2\right] E_{z} ; \mathbf{r} ; \mathbf{0}\right)=\frac{1}{\sqrt{\pi} E_{z} a_{z}^{3}} \exp \left(-\frac{z^{2}}{2 a_{z}^{2}}\right) \sum_{j=0}^{\infty} \frac{(-1)^{j}}{2^{2 j} j !} H_{2 j}\left(z / a_{z}\right) \mathcal{G}^{2 \mathrm{D}}\left(\left[\frac{\epsilon_{\boldsymbol{\lambda}}-2 j}{\eta}+1\right] E_{\rho} ; \rho, 0\right) .
$$

This expression is equivalent to Eq. (28) but converges faster for pancake-shaped traps than Eq. (28). Using Eq. (37) in Eq. (20), we obtain

$$
\begin{array}{r}
I_{\boldsymbol{\lambda}, \lambda^{\prime}}^{3 \mathrm{D}}\left(\epsilon_{\boldsymbol{\lambda}^{\prime}}\right)=2 \sqrt{\pi}(-1)^{m} \delta_{m, m^{\prime}} \times \\
\lim _{j_{\max } \rightarrow \infty} \sum_{j=0}^{j_{\max }} \frac{(-1)^{j} \sqrt{\pi^{1 / 2}(2 j) !}}{2^{j} j !} I_{n_{z}, n_{z}^{\prime}}^{\mathrm{p}}(j) I_{n_{\rho}, n_{\rho}^{\prime}, m}^{\mathrm{p}}\left(\epsilon_{\boldsymbol{\lambda}^{\prime}}, j\right),
\end{array}
$$

where

$$
I_{n_{z}, n_{z}^{\prime}}^{\mathrm{p}}(j)=a_{z}^{1 / 2} \int_{-\infty}^{\infty} \varphi_{2 j}\left(\frac{\sqrt{3}}{2} z\right) \varphi_{n_{z}^{\prime}}\left(-\frac{z}{2}\right) \varphi_{n_{z}}(z) d z
$$




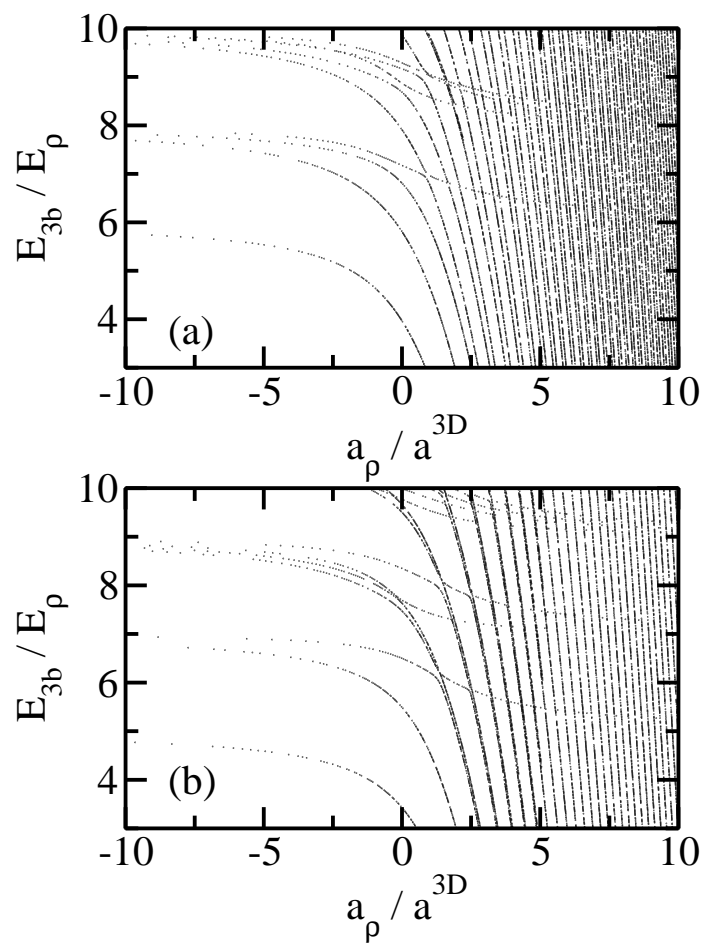

FIG. 5: Relative three-body energies $E_{3 \mathrm{~b}} / E_{\rho}$ as a function of the inverse scattering length $a_{\rho} / a^{3 \mathrm{D}}$ for a pancake-shaped trap with aspect ratio $\eta=1 / 2$ and (a) $M=0$ and $\Pi_{z}=+1$, and (b) $M= \pm 1$ and $\Pi_{z}=+1$.

and

$$
\begin{aligned}
& I_{n_{\rho}, n_{\rho}^{\prime}, m}^{\mathrm{P}}\left(\epsilon_{\boldsymbol{\lambda}^{\prime}}, j\right)= \\
& E_{z} a_{z}^{2} \int_{0}^{\infty} \mathcal{G}^{2 \mathrm{D}}\left(\left[\frac{\epsilon_{\boldsymbol{\lambda}^{\prime}}-2 j}{\eta}+1\right] E_{\rho} ; \frac{\sqrt{3}}{2} \rho ; 0\right) R_{n_{\rho}^{\prime}, m}\left(\frac{\rho}{2}\right) R_{n_{\rho}, m}(\rho) \rho d \rho .
\end{aligned}
$$

Details regarding the evaluation of the integrals are explained in Appendix A. In the following, we limit ourselves to cases where the reciprocal of the aspect ratio is an integer. In this case, we have [6, 7]

$$
\mathcal{F}^{3 \mathrm{D}}\left(\epsilon_{\boldsymbol{\lambda}}, \eta\right)=-2 \eta \sum_{k=0}^{1 / \eta-1} \frac{\Gamma\left(-\frac{\epsilon_{\boldsymbol{\lambda}}}{2}+k \eta\right)}{\Gamma\left(-\frac{\epsilon_{\boldsymbol{\lambda}}+1}{2}+k \eta\right)} .
$$

Figure 5 shows the relative three-body energies $E_{3 \mathrm{~b}} / E_{\rho}$ as a function of the inverse scattering length for $\eta=1 / 2$, $\Pi_{z}=+1$, and (a) $M=0$ and (b) $M= \pm 1$. In the $\left(a^{3 \mathrm{D}}\right)^{-1} \rightarrow-\infty$ limit, the ground state has $M= \pm 1$ and $\Pi_{z}=+1$ symmetry. In the $\left(a^{3 \mathrm{D}}\right)^{-1} \rightarrow+\infty$ limit, in contrast, the ground state has $M=0$ and $\Pi_{z}=+1$ symmetry.

Figure [6] shows the relative energy of the energetically lowest-lying state, the so-called crossover curve, of the threebody system with $\Pi_{z}=+1$ for various aspect ratios of the trap $(\eta=1 / 2, \cdots, 1 / 10)$ as a function of the inverse scattering length $a_{\rho} / a^{3 \mathrm{D}}$. For comparative purposes, we subtract the relative ground state energy of $E_{z}$ of the noninteracting one-dimensional system, that is, the energy that the system would have in the $z$-direction if the dynamics in the tight confinement direction were frozen, from the full three-dimensional energy. The scattering lengths at which the symmetry of the corresponding eigenstate changes from $M= \pm 1$ to $M=0$ are marked by asterisks. The symmetry change occurs around $a_{z} / a^{3 \mathrm{D}} \approx 1$ (see inset).

It is instructive to compare Fig. 6 (pancake-shaped trap) and Fig. 2 (cigar-shaped trap). For both geometries, the crossover curve changes symmetry. The change of the symmetry is associated with the low-energy coordinate (the $\rho$-coordinate for pancake-shaped systems and the $z$-coordinate for cigar-shaped systems). For both geometries, the symmetry change occurs, for the aspect ratios considered, when $a^{3 \mathrm{D}}$ is of the order of the oscillator length in the tight confinement direction. 


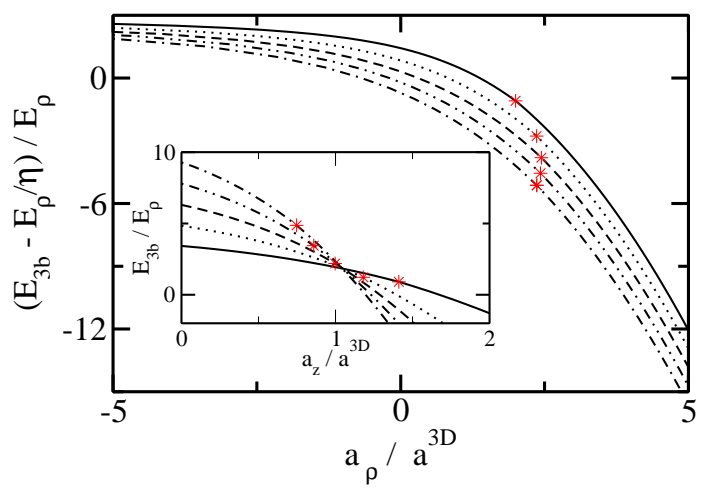

FIG. 6: (Color online) "Crossover curve" of the three-body system with $\Pi_{z}=+1$, shifted by $E_{z}=E_{\rho} / \eta$, for various aspect ratios of the trap, $\eta=1 / 2$ (solid line), $1 / 4$ (dotted line), 1/6 (dashed line), $1 / 8$ (dash-dot-dotted line), and $1 / 10$ (dash-dotted line) as a function of $a_{\rho} / a^{3 \mathrm{D}}$. The scattering lengths at which the $M$ quantum number of the corresponding eigenstate changes from $M= \pm 1$ ("left side of the graph") to $M=0$ ("right side of the graph") are marked by asterisks. The inset shows the (unshifted) crossover curve as a function of $a_{z} / a^{3 \mathrm{D}}$.

Next, we consider the small $\eta$ limit in more detail. For $\eta \ll 1$ and $|\epsilon| \ll 1$, we have [․, 7]

$$
\left.\mathcal{F}^{3 \mathrm{D}}(\epsilon, \eta)\right|_{\eta \ll 1} \approx \frac{1}{\sqrt{\pi}}\left[2 \mathcal{F}^{2 \mathrm{D}}(\epsilon)-2 \ln (\mathcal{C})-\ln (\eta)\right],
$$

and the two-body eigenequation for the relative energy becomes

$$
\mathcal{F}^{2 \mathrm{D}}(\epsilon)=\ln \left(a_{\text {ren }}^{2 \mathrm{D}} / a_{\rho}\right)
$$

where the renormalized two-dimensional scattering length $a_{\mathrm{ren}}^{2 \mathrm{D}}$ is given by [22]

$$
\frac{a_{\mathrm{ren}}^{2 \mathrm{D}}}{a_{\rho}}=\sqrt{\eta} \mathcal{C} \exp \left(-\frac{\sqrt{\pi} a_{z}}{2 a^{3 \mathrm{D}}}\right)
$$

with $\mathcal{C} \approx 1.479$. Figure 7 (a) shows the relative two-body energies for a system with $\eta=1 / 10, M=0$ and $\Pi_{z}=$ +1 obtained by solving the eigenequation $\mathcal{F}^{3 \mathrm{D}}(\epsilon, \eta=1 / 10)=-a_{z} / a^{3 \mathrm{D}}$ [see Eq. (41) for $\mathcal{F}^{3 \mathrm{D}}(\epsilon, \eta)$ ]. Figure 7 (b) compares the full three-dimensional energy (solid line) with the energy obtained by solving the strictly two-dimensional eigenequation, Eq. (43), with renormalized two-dimensional scattering length $a_{\mathrm{ren}}^{2 \mathrm{D}}$ (dotted line). For comparative purposes, we add the energy of the tight confinement direction to the energy of the strictly two-dimensional system. The inset of Fig. Z(b) shows the difference between the strictly two-dimensional energy and the full three-dimensional energy as a function of $a_{\rho} / a^{3 \mathrm{D}}$. The maximum deviation occurs around unitarity and is of the order of $0.4 \%$.

To treat the three-body system in the small $\eta$ limit, we insert Eqs. (42) and (44) into Eq. (19). This yields

$$
\sum_{\boldsymbol{\lambda}^{\prime}}\left[\frac{\sqrt{\pi}}{2} I_{\boldsymbol{\lambda}, \boldsymbol{\lambda}^{\prime}}^{3 \mathrm{D}}\left(\epsilon_{\boldsymbol{\lambda}^{\prime}}\right)-\mathcal{F}^{2 \mathrm{D}}\left(\epsilon_{\boldsymbol{\lambda}^{\prime}}\right) \delta_{\boldsymbol{\lambda}, \boldsymbol{\lambda}^{\prime}}\right] f_{\boldsymbol{\lambda}^{\prime}}=\ln \left(\frac{a_{\rho}}{a_{\mathrm{ren}}^{2 \mathrm{D}}}\right) f_{\boldsymbol{\lambda}}
$$

For fixed $M$, Eq. (45) reduces to the strictly two-dimensional eigenequation, Eq. (25), if (i) the sum over $\boldsymbol{\lambda}^{\prime}$ is restricted to a sum over $n_{\rho}^{\prime}$ [i.e., if $\boldsymbol{\lambda}^{\prime}=\left(0, n_{\rho}^{\prime}, m^{\prime}=M\right)$ ]; (ii) the index $\boldsymbol{\lambda}$ is restricted to $\boldsymbol{\lambda}=\left(0, n_{\rho}, m=M\right)$; (iii) the energy $E_{3 \mathrm{~b}}$ is replaced by $E_{3 \mathrm{~b}}-E_{z}$; and (iv) $j_{\max }$ in Eq. (38) is set to zero. Under these assumptions, Eq. (45) reduces to Eq. (25) with $a^{2 \mathrm{D}}$ replaced by $a_{\text {ren }}^{2 \mathrm{D}}$.

Figure 8 (a) shows the relative three-body energies for states with $M=0$ and $\Pi_{z}=+1$ as a function of the inverse scattering length $a_{\rho} / a^{3 \mathrm{D}}$ for a pancake-shaped trap with $\eta=1 / 10$. Figure 8 (b) compares the energy of the energetically lowest-lying three-atom state with $M=0$ (solid line) [see thick solid line in Fig. 8(a)] with the corresponding state obtained by solving the strictly two-dimensional equation with renormalized two-dimensional scattering length $a_{\text {ren }}^{2 \mathrm{D}}$ (dotted line). The inset shows the difference between the energies obtained within the strictly two-dimensional and the full three-dimensional frameworks. Similar to the one-dimensional case, the maximum of the deviation occurs near unitarity. Comparison of the insets of Figs. $7(b)$ and $8(b)$ suggests that, at least in this low-energy example, the presence of the third atom does not, in a significant manner, reduce the applicability of the strictly two-dimensional framework. For the same aspect ratio, the deviations are expected to increase with increasing energy. 


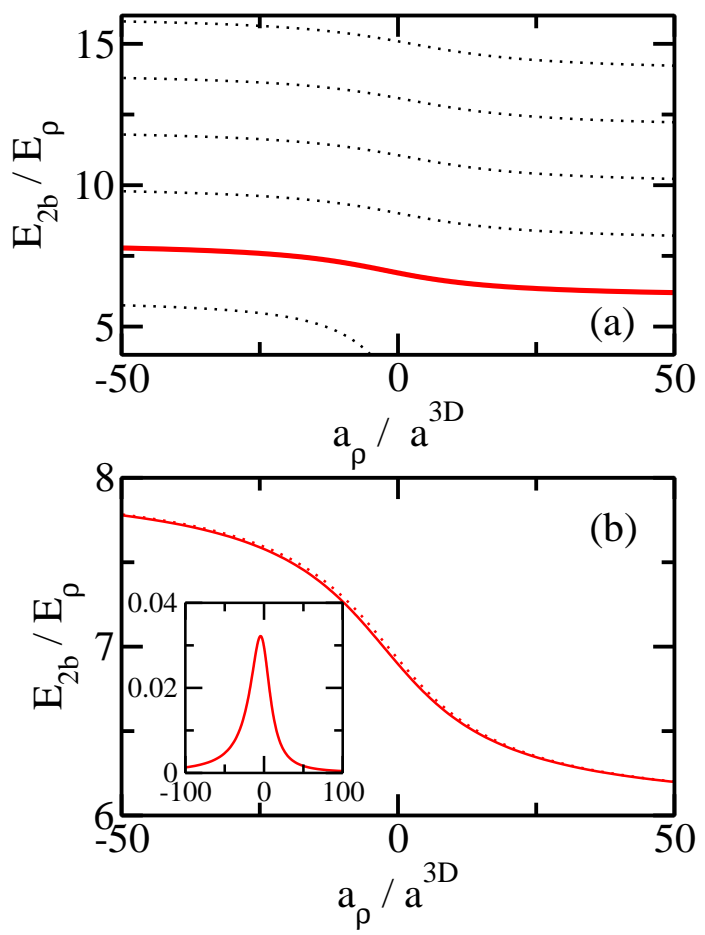

FIG. 7: (Color online) (a) Relative two-body energies $E_{2 \mathrm{~b}} / E_{\rho}$ as a function of the inverse scattering length $a_{\rho} / a^{3 \mathrm{D}}$ for a pancake-shaped trap with aspect ratio $\eta=1 / 10, M=0$ and $\Pi_{z}=+1$. (b) The solid curve from panel (a) is replotted and compared with the energy obtained by solving the strictly two-dimensional eigenequation with renormalized two-dimensional scattering length $a_{\mathrm{ren}}^{2 \mathrm{D}}$ (dotted line; the energy $E_{z} / 2$ has been added to allow for a comparison with the full three-dimensional energy). The inset shows the difference between the dotted and solid lines as a function of the inverse scattering length $a_{\rho} / a^{3 \mathrm{D}}$. The scale of the $y$-axis is identical to that of the inset of Fig. 8 (b).

\section{SECOND- AND THIRD-ORDER VIRIAL COEFFICIENTS}

This section utilizes the two- and three-body energy spectra to determine the second- and third-order virial coefficients as functions of the $s$-wave scattering length $a^{3 \mathrm{D}}$, aspect ratio $\eta$ and temperature $T$. The $n^{\text {th }}$-order virial coefficient $b_{n}$ enters into the high-temperature expansion of the grand-canonical thermodynamic potential $\Omega$ of the equal-mass two-component Fermi gas with interspecies $s$-wave interactions [11 19], $\Omega=\Omega^{(1)}+\Omega^{(2)}+\Omega^{(12)}$, where $\Omega^{(1)}$ and $\Omega^{(2)}$ denote the grand-canonical thermodynamic potential of the spin-up component and the spin-down component, respectively, and $\Omega^{(12)}$ accounts for the interspecies interactions,

$$
\Omega^{(12)}=-k_{\mathrm{B}} T Q_{1,0} \sum_{n=2}^{\infty} b_{n} z^{n} .
$$

Here, $z$ is the fugacity, $z=\exp \left[\mu /\left(k_{\mathrm{B}} T\right)\right]$. In the high-temperature limit, $z$ is a small parameter and the expansion given in Eq. (46) is expected to provide a good description if the sum is terminated at quadratic or cubic order. The coefficient $b_{2}$ of the $z^{2}$ term is determined by one- and two-body physics and the coefficient $b_{3}$ of the $z^{3}$ term is determined by one-, two- and three-body physics. As the temperature approaches the transition temperature from above, the de Broglie wave length increases and, correspondingly, the fugacity $z$ increases. It follows that the number of $b_{n}$ coefficients needed to accurately describe the thermodynamics increases with decreasing temperature. Comparison with experimental data has shown that the inclusion of $b_{2}$ and $b_{3}$ yields quite accurate descriptions of the high-temperature thermodynamics of $s$-wave interacting two-component Fermi gases (see, e.g., Refs. [14, 17, 18]).

In Eq. (46), $Q_{1,0}$ denotes the canonical partition function of a single spin-up particle. We define the canonical 


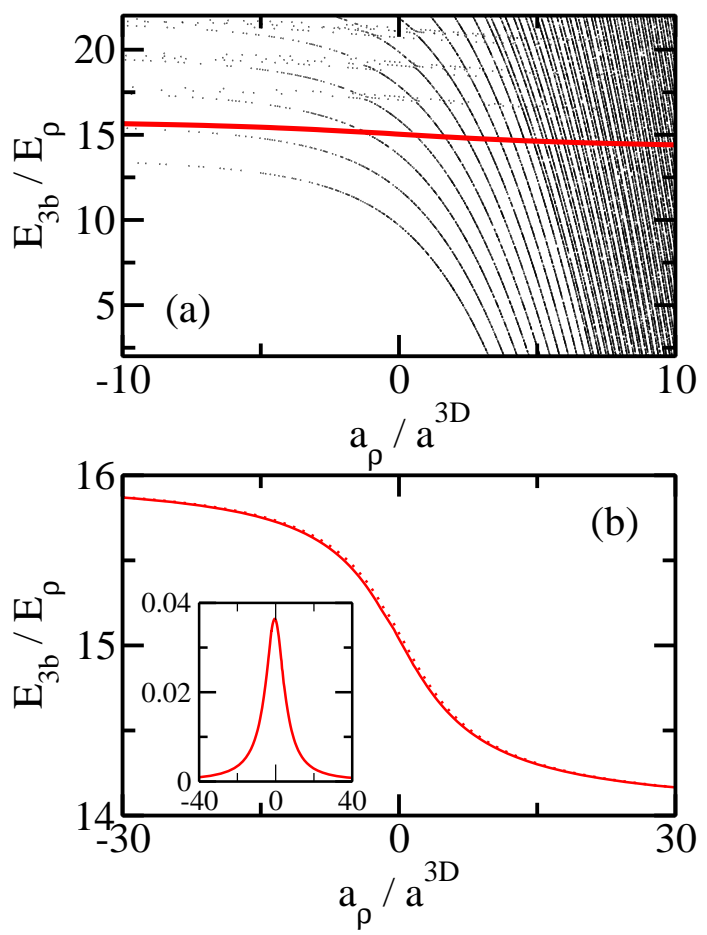

FIG. 8: (Color online) (a) Relative three-body energies $E_{3 \mathrm{~b}} / E_{\rho}$ as a function of the inverse scattering length $a_{\rho} / a^{3 \mathrm{D}}$ for a pancake-shaped trap with aspect ratio $\eta=1 / 10, M=0$ and $\Pi_{z}=+1$. (b) The solid curve from panel (a) is replotted and compared with the energy obtained by solving the strictly two-dimensional eigenequation with renormalized two-dimensional scattering length $a_{\mathrm{ren}}^{2 \mathrm{D}}$ (dotted line; the energy of $E_{z}$ has been added to allow for a comparison with the full three-dimensional energy). The inset shows the difference between the dotted and solid lines as a function of the inverse scattering length $a_{\rho} / a^{3 \mathrm{D}}$.

partition function $Q_{n_{1}, n_{2}}$ of the system consisting of $n_{1}$ spin-up particles and $n_{2}$ spin-down particles through

$$
Q_{n_{1}, n_{2}}=\sum_{j} \exp \left(-\frac{E_{j}^{\mathrm{tot}}\left(n_{1}, n_{2}\right)}{k_{\mathrm{B}} T}\right),
$$

where $E_{j}^{\text {tot }}\left(n_{1}, n_{2}\right)$ denotes the total energy of the system (including the center-of-mass energy) and the summation over $j$ includes all quantum numbers allowed by the symmetry of the system. For equal-mass fermions, as considered throughout this paper, we have $Q_{1,0}=Q_{0,1}=Q_{1}$. The virial coefficients $b_{2}$ and $b_{3}$ can be expressed as [14, 41]

$$
b_{2}=\frac{Q_{1,1}-Q_{1}^{2}}{Q_{1}}
$$

and

$$
b_{3}=2 \frac{Q_{2,1}-Q_{2,0} Q_{1}-b_{2} Q_{1}^{2}}{Q_{1}}
$$

The virial coefficients $b_{2}$ and $b_{3}$ depend on the interspecies scattering length $a^{3 \mathrm{D}}$, aspect ratio $\eta$ and temperature $T$. Once the thermodynamic potential is known, physical observables such as the pressure and the entropy can be calculated.

We now discuss the determination of $b_{2}$ and $b_{3}$ for equal-mass two-component Fermi gases under anisotropic harmonic confinement. In this case, the single-particle canonical partition function $Q_{1}$ can be determined analytically,

$$
Q_{1}=\frac{\exp \left([1 / 2+\eta] \tilde{\omega}_{z}\right)}{\left[\exp \left(\tilde{\omega}_{z}\right)-1\right]\left[\exp \left(\eta \tilde{\omega}_{z}\right)-1\right]^{2}},
$$


where $\tilde{\omega}_{z}$ denotes the "inverse temperature" in units of $E_{z}, \tilde{\omega}_{z}=E_{z} /\left(k_{\mathrm{B}} T\right)$. Alternatively (see below), we express the inverse temperature in units of $E_{\rho}$ or $E_{\text {ave }}, \tilde{\omega}_{\rho}=E_{\rho} /\left(k_{\mathrm{B}} T\right)$ and $\tilde{\omega}_{\text {ave }}(\eta)=E_{\text {ave }} /\left(k_{\mathrm{B}} T\right)$. The average energy $E_{\text {ave }}$ is defined in terms of the root-mean-square or, in short, average angular frequency $\omega_{\text {ave }}(\eta), E_{\text {ave }}=\hbar \omega_{\text {ave }}(\eta)$, where

$$
\omega_{\text {ave }}(\eta)=\sqrt{\frac{2 \omega_{\rho}^{2}+\omega_{z}^{2}}{3}} .
$$

We note that the average angular frequency coincides with the angular trapping frequency for $\eta=1$ but not for $\eta \neq 1$. Below, we frequently suppress the explicit dependence of $\tilde{\omega}_{\text {ave }}$ on $\eta$. The partition functions $Q_{1,1}$ and $Q_{2,1}$ can be determined from the two- and three-body energy spectra (see Secs. III and [V] for each $s$-wave scattering length $a^{3 \mathrm{D}}$, aspect ratio $\eta$ and temperature $T$.

At unitarity, the high-temperature expansion of $b_{n}$ reads (for $\eta=1$, see Ref. [14])

$$
b_{n} \approx b_{n}^{(0)}+b_{n}^{(2)}(\eta) \tilde{\omega}_{z}^{2}+b_{n}^{(4)}(\eta) \tilde{\omega}_{z}^{4}+\cdots
$$

The coefficients $b_{n}^{(0)}$, that is, the high-temperature limits of the trapped virial coefficients $b_{n}$, are independent of the aspect ratio $\eta$. This has previously been shown to be the case for $n=2$ [42]. Here, we extend the argument to all $n$. Application of the local density approximation [14, 19, 43] to axially symmetric confinement potentials shows that the virial coefficients $b_{n}^{\text {hom }}$ of the homogeneous system, which have been shown to be temperature-independent at unitarity [11 13$]$, are related to $b_{n}^{(0)}$ through

$$
b_{n}^{\text {hom }}=n^{3 / 2} b_{n}^{(0)}
$$

Since Eq. (53) holds for all $\eta, b_{n}^{(0)}$ has to be independent of $\eta$ for all $n$.

The expansion coefficients $b_{n}^{(k)}, k=2,4, \cdots$, parametrize "trap corrections", that is, corrections that arise due to the fact that the harmonic confinement defines a meaningful (finite) length scale. In fact, for $\eta \neq 1$, the confinement defines two length scales, suggesting that the $b_{n}^{(k)}, k=2,4, \cdots$, depend on $\eta$. Equation (52) expresses the temperature dependence of $b_{n}$ in terms of the inverse temperature associated with the $z$-direction, regardless of whether $\eta$ is greater or smaller than 1. Interestingly, it was shown in Ref. [42] that the dependence of $b_{2}^{(k)}, k=2,4, \cdots$, on the aspect ratio can be parametrized, to a good approximation, in terms of the average inverse temperature $\tilde{\omega}_{\text {ave }}(\eta)$,

$$
b_{2}^{(k)}(\eta) \tilde{\omega}_{z}^{k} \approx b_{2}^{(k)}(1)\left[\tilde{\omega}_{\text {ave }}(\eta)\right]^{k} .
$$

Equation (54) implies that the trap corrections for two-body systems with $\eta \neq 1$ can be parametrized in terms of the trap corrections for the spherically symmetric system if the inverse temperature is expressed in terms of the average trapping frequency that characterizes the anisotropic system.

We now illustrate that Eq. (54) applies not only to $b_{2}$ but also to $b_{3}$. Figures 9(a) and 9)(b) show the third-order virial coefficient at unitarity for systems with $\eta \geq 1$ and $\eta \leq 1$, respectively. The virial coefficients are plotted as a function of the inverse temperature expressed in units of the weak confinement direction, i.e., in terms of $\omega_{z}$ for $\eta \geq 1$ and in terms of $\omega_{\rho}$ for $\eta \leq 1$. In the high-temperature limit, $b_{3}$ approaches a constant, confirming that $b_{3}^{(0)}$ is independent of $\eta$. The dotted lines show the third-order virial coefficient for $\eta=1$, calculated using the average trapping frequency characteristic for the respective anisotropic system. Figure 9 illustrates that the third-order virial coefficient for anisotropic traps is approximated well by that for $\eta=1$ with appropriately scaled angular frequency. The insets of Fig. 9] show that the third-order virial coefficients of the anisotropic system collape, to a very good approximation, to a universal curve over a surprisingly large temperature regime, i.e., down to temperatures around $k_{\mathrm{B}} T \approx E_{\text {ave }} / 2$. We conjecture that $b_{n}^{(k)}(\eta) \tilde{\omega}_{z}^{k}$ can be approximated quite well by $b_{n}^{(k)}(1)\left[\tilde{\omega}_{\text {ave }}(\eta)\right]^{k}$ for $n=4,5, \cdots$ as well, as long as $k$ is not too large, i.e, as long as the temperature is not too low.

Next, we discuss the behavior of $b_{2}$ for finite $s$-wave scattering lengths. Figure 10 shows a surface plot of $b_{2}$ for $\eta=1$ as a function of the inverse scattering length $a_{\text {ave }} / a^{3 \mathrm{D}}\left(a^{3 \mathrm{D}} \leq 0\right)$ and the inverse temperature $\tilde{\omega}_{\text {ave }}$. Here, $a_{\text {ave }}$ denotes the oscillator length associated with the average trapping frequency, $a_{\text {ave }}=\sqrt{\hbar /\left(\mu \omega_{\text {ave }}\right)}$. At unitarity, $b_{2}$ is only weakly-dependent on the temperature and approximately equal to $1 / 2$ (see discussion above). The smallest inverse temperature $\tilde{\omega}_{\text {ave }}$ considered in Fig. 10 is 0.0003 . For this inverse temperature, $b_{2}$ is fairly close to $1 / 2$ for all $a_{\text {ave }} / a^{3 D}$ shown. Thus, Fig. 10 shows that the high-temperature limit of $b_{2}$ is nearly independent of the scattering length. This behavior can, as we now show, be understood from the two-body energy spectrum.

Figure 11(a) shows selected relative two-body energies as a function of $a_{\text {ave }} / a^{3 \mathrm{D}}$ for the trapped system with $\eta=1$. In the low-energy regime (solid line), the two-body energy changes by nearly $2 E_{\text {ave }}$ for the scattering length range shown. As the energy increases (dashed and dotted lines show energies around $200 E_{\text {ave }}$ and $20000 E_{\text {ave }}$, respectively), 

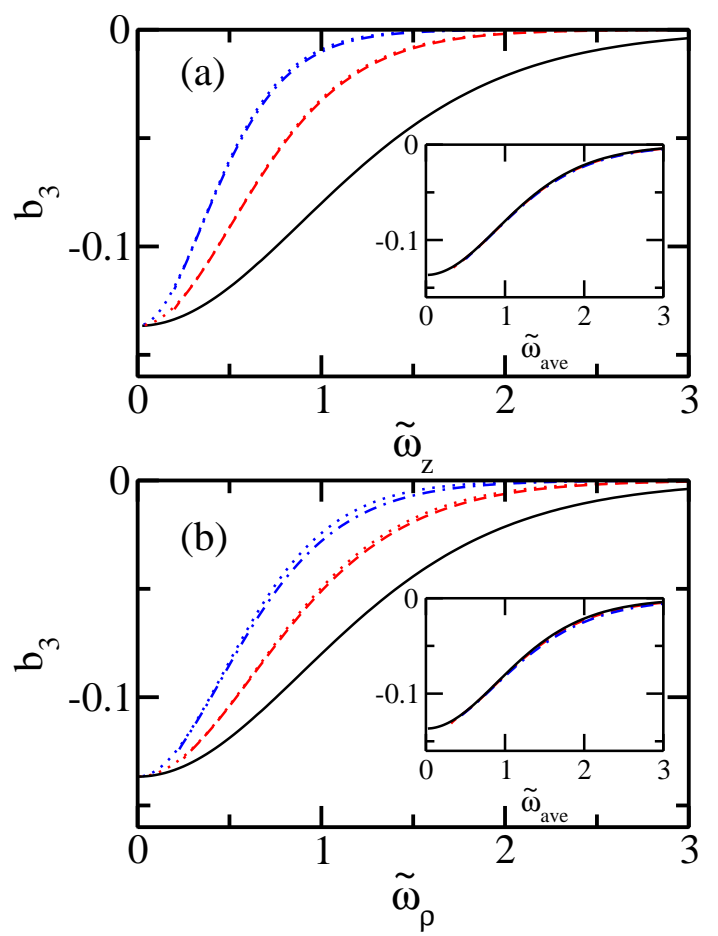

FIG. 9: (Color online) (a) Third-order virial coefficient $b_{3}$ at unitarity as a function of the inverse temperature $\tilde{\omega}_{z}$ for $\eta=1$ (solid line), 2 (dashed line), and 3 (dash-dotted line). (b) Third-order virial coefficient $b_{3}$ at unitarity as a function of the inverse temperature $\tilde{\omega}_{\rho}$ for $\eta=1$ (solid line), $1 / 2$ (dashed line), and $1 / 3$ (dash-dotted line). For $\eta \neq 1, b_{3}$ terminates at the inverse temperature of about 0.25 since our calculations include a finite number of three-body energies; obtaining the behavior of $b_{3}$ in the high-temperature limit requires the inclusion of infinitely many three-body energies. For $\eta \neq 1$, dotted lines show $b_{3}$ for $\eta=1$, calculated using the average frequency of the respective anisotropic system. This approximate description is quite good. The insets of panels (a) and (b) show the same data as the main figure, but now as a function of $\tilde{\omega}_{\text {ave }}$ as opposed to $\tilde{\omega}_{z}$ and $\tilde{\omega}_{\rho}$. The insets show that the third-order virial coefficients for different $\eta$ collapse to a universal curve for all $\eta$ (deviations arise in the low-temperature regime, i.e., for $\left.\tilde{\omega}_{\text {ave }} \gtrsim 1\right)$.

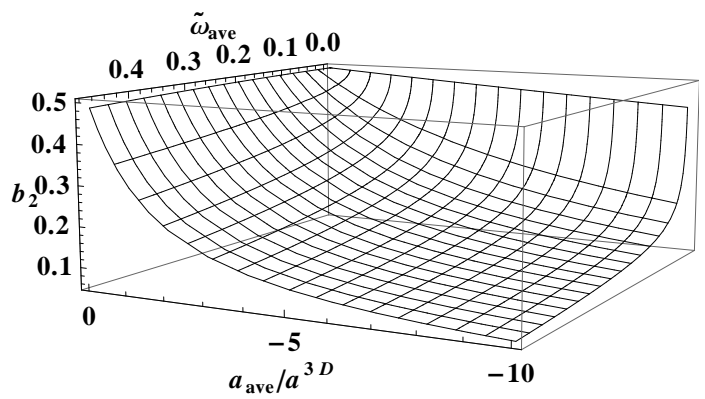

FIG. 10: Second-order virial coefficient $b_{2}$ for the two-body system under isotropic confinement as a function of the inverse

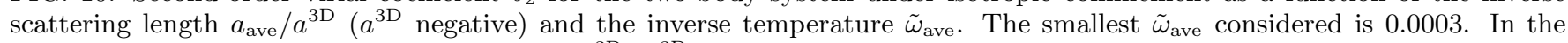
$\tilde{\omega}_{\text {ave }} \rightarrow 0$ limit, $b_{2}$ approaches $1 / 2$ for all $a_{\text {ave }} / a^{3 \mathrm{D}}$ ( $a^{3 \mathrm{D}}<0$; see text for further discussion). 

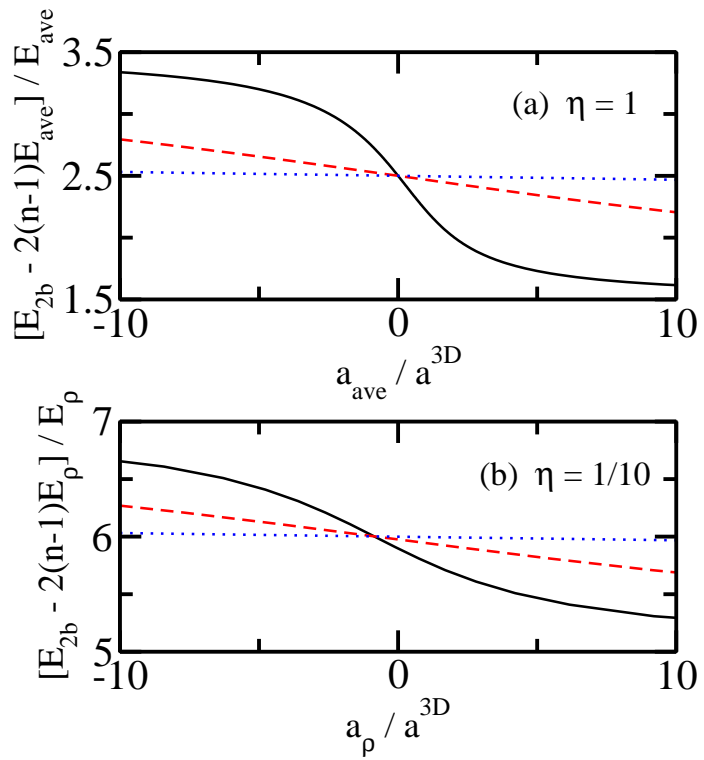

FIG. 11: (Color online) (a) Relative two-body energies $E_{2 \mathrm{~b}} / E_{\text {ave }}$, shifted by $2(n-1)$, for isotropic confinement $(\eta=1$ and $s$-wave channel) as a function of the inverse scattering length $a_{\text {ave }} / a^{3 \mathrm{D}}$. (b) Relative two-body energies $E_{2 \mathrm{~b}} / E_{\rho}$, shifted by $2(n-1)$, for pancake-shaped confinement $\left(\eta=1 / 10, M=0\right.$, and $\left.\Pi_{z}=+1\right)$ as a function of the inverse scattering length $a_{\rho} / a^{3 \mathrm{D}}$. Solid, dotted and dashed lines show the energies for $n=1,100$ and 10000, respectively.

the two-body energy undergoes less of a change and eventually becomes nearly flat over the scattering length region shown. This implies that the high-energy portion of the two-body spectrum looks like that of the unitary gas over an increasingly large region around unitarity. The behavior of the energy spectrum can be understood by expanding the transcendental two-body eigenequation $\mathcal{F}^{3 \mathrm{D}}(\epsilon, 1)=-a_{\text {ave }} / a^{3 \mathrm{D}}$ around unitarity. Assuming $\left|a_{\text {ave }} / a^{3 \mathrm{D}}\right| \ll 1$, we find [42, 44]

$$
\frac{E_{2 \mathrm{~b}}}{E_{\mathrm{ave}}}-\left(2 n+\frac{1}{2}\right) \approx-\frac{\Gamma(n+1 / 2)}{\pi \Gamma(n+1)} \frac{a_{\mathrm{ave}}}{a^{3 \mathrm{D}}}
$$

Equation (55) provides a good description of the energies as long as the right hand side is small. Since the right hand side of Eq. (55) scales for large $n$ as $\left(a_{\text {ave }} / a^{3 \mathrm{D}}\right) / \sqrt{n}$, Eq. (55) provides, as $n$ increases, a good description for an increasingly large region around unitarity. That is, the energies vary approximately linearly with $a_{\text {ave }} / a^{3 \mathrm{D}}$, with a slope that approaches zero, as $n \rightarrow \infty$. This analysis rationalizes why $b_{2}$ approaches $1 / 2$ as $T \rightarrow \infty$, regardless of the value of the scattering length $\left(a^{3 \mathrm{D}}<0\right.$ and finite).

Figure 10 has been obtained for a spherically symmetric system, that is, for $\eta=1$. We now demonstrate that Fig. 10 applies, for experimentally relevant temperatures, to all aspect ratios and not just to $\eta=1$. Figure 12 shows $b_{2}$ as a function of $\tilde{\omega}_{\text {ave }}$ for three different aspect ratios, $\eta=1$ (solid lines), $\eta=1 / 5$ (dashed lines) and $\eta=1 / 100$ (dotted lines). Three different scattering lengths are considered: $a^{3 \mathrm{D}}=-a_{\text {ave }}, \infty$ and $a_{\text {ave }}$. It can be seen that $b_{2}$ is, to a very good approximation, independent of $\eta$ in the high-temperature (small $\tilde{\omega}_{\text {ave }}$ ) limit. We stress that the independence of $b_{2}$ of $\eta$ requires that the inverse temperature and scattering length are expressed in terms of the average oscillator units $E_{\text {ave }}$ and $a_{\text {ave }}$, respectively.

To understand the universality implied by Fig. [12, that is, the fact that Fig. 10] applies to all aspect ratios and not just to $\eta=1$, we analyze the behavior of the high-lying part of the relative two-body spectra for $\eta \neq 1$. Figure 11(b) 


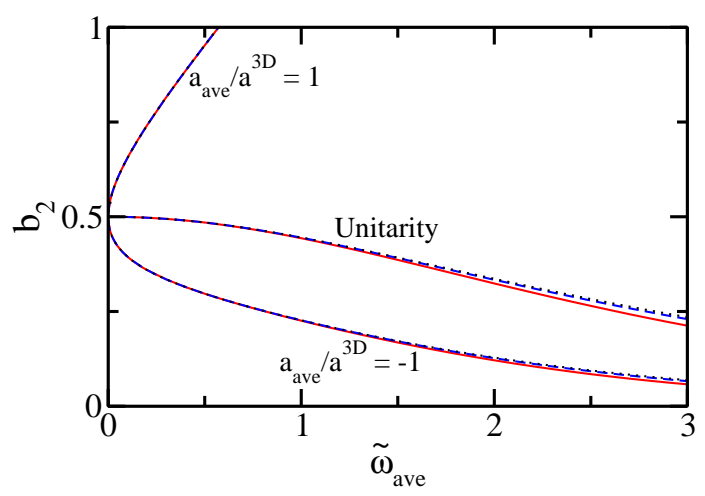

FIG. 12: (Color online) Second-order virial coefficient $b_{2}$ of the trapped two-body system as a function of the inverse temperature $\tilde{\omega}_{\text {ave }}$ for three different values of the inverse scattering length $\left(a_{\text {ave }} / a^{3 \mathrm{D}}=-1,0\right.$ and +1 ; see labels $)$ for $\eta=1$ (solid lines), $\eta=1 / 5$ (dashed lines) and $\eta=1 / 100$ (dotted lines).

exemplarily shows the relative energies for a pancake-shaped system with $\eta=1 / 10$. Comparison with Fig. 11)(a) shows that the qualitative behavior of the high-energy part of the spectrum is independent of $\eta$. This is confirmed by a more quantitative analysis that Taylor expands the implicit eigenequation for the anisotropic two-body system around unitarity. We conclude that two two-body systems with different aspect ratios but identical $a_{\text {ave }} / a^{3 \mathrm{D}}$ and $\tilde{\omega}_{\text {ave }}$ are characterized by approximately the same $b_{2}$ value. Our analysis of the three-body energies for anisotropic confinement suggests that analogous conclusions hold for $b_{3}$. We speculate that the conclusions hold also for the virial coefficients with $n>3$.

To estimate the extent of the universal behavior, it is instructive to reexpress $\tilde{\omega}_{\text {ave }}$ in terms of the Fermi temperature. For a spin-balanced system of $N$ fermions under spherically symmetric confinement, we use the semi-classical expression $k_{\mathrm{B}} T_{\mathrm{F}}=(3 N)^{1 / 3} \hbar \omega_{\text {ave }}$, yielding $\tilde{\omega}_{\text {ave }}=(3 N)^{-1 / 3}\left(T / T_{\mathrm{F}}\right)^{-1}$. For $N=10^{2}, 10^{4}$ and $10^{6}, T / T_{\mathrm{F}}=1$ corresponds to $\tilde{\omega}_{\text {ave }} \approx 0.149,0.032$ and 0.007 , respectively. For these temperatures, the thermodynamic behavior is, according to our discussion above, expected to be essentially universal over a fairly wide range of scattering lengths. For $T / T_{\mathrm{F}}=1$, we estimate that the deviation of $b_{2}$ from the value $1 / 2$ approaches $5 \%$ for $a_{\text {ave }} / a^{3 \mathrm{D}} \approx-0.16,-0.38$ and -0.78 for $N=10^{2}, 10^{4}$ and $10^{6}$, respectively. This implies that uncertainties of the scattering length dependence on the magnetic field in recent experiments at unitarity [17, 18] should have a negligibly small effect on the equation of state at unitarity. For all three cases considered above, the corresponding $\left(k_{\mathrm{F}} a^{3 \mathrm{D}}\right)^{-1}$ values is approximately -0.03 .

The determination of the high-temperature behavior of the third-order virial coefficient for different scattering lengths and aspect ratios is much more demanding than that of the second-order virial coefficient. Although our analysis of $b_{3}$ is less exhaustive than that of $b_{2}$, it suggests that the conclusions drawn above for the second-order virial coefficient carry over to the third-order virial coefficient.

\section{CONCLUSION}

This paper developed a Lippmann-Schwinger equation based approach to determine the energy spectrum and corresponding eigenstates of three-body systems with zero-range $s$-wave interactions under harmonic confinement with different transverse and longitudinal angular trapping frequencies. The formalism was applied to the equal-mass system consisting of two identical fermions and a third distinguishable particle in a different spin-state. The energy spectra were determined as a function of the interspecies $s$-wave scattering length for various aspect ratios $\eta, \eta>1$ (cigar-shaped trap) and $\eta<1$ (pancake-shaped trap). For $\eta \gg 1$, we showed that the low-energy portion of the energy spectra are reproduced well by an effective one-dimensional Hamiltonian with renormalized one-dimensional coupling constant. Similarly, for $\eta \ll 1$, we showed that the low-energy portion of the energy spectra are reproduced well by an effective two-dimensional Hamiltonian with renormalized two-dimensional coupling constant. As the energy increases, the description based on these effective low-dimensional Hamiltonian deteriorates.

The two- and three-body energy spectra were then used to determine the second- and third-order virial coefficients that determine the virial equation of state, applicable to two-component Fermi gases at temperatures above the Fermi temperature. Our key findings are: 
(i) At unitarity, the second- and third-order virial coefficients $b_{2}$ and $b_{3}$ approach constants in the high-temperature regime. The constants (referred to as $b_{2}^{(0)}$ and $b_{3}^{(0)}$ ) are independent of $\eta$.

(ii) For finite scattering length $a^{3 \mathrm{D}}$, we find that $b_{2}$ and $b_{3}$ collapse, to a very good approximation, to a single curve for all $\eta$ if the temperature and scattering length are expressed in terms of the average energy $E_{\text {ave }}$ and the average oscillator length $a_{\text {ave }}$, respectively. Deviations from the universal curve arise in the low-temperature regime where the virial equation of state is not applicable.

(iii) The virial coefficient $b_{2}$ is approximately equal to $1 / 2$ over a fairly large temperature and scattering length regime around unitarity.

The work presented in this paper is directly relevant to on-going cold atom experiments. The three-body spectra, e.g., can be measured experimentally through rf spectroscopy [23, 45, 46]. Moreover, the formalism can be employed to characterize the molecular states in more detail, quantifying the "perturbation" of the dimer due to the third particle throughout the dimensional crossover. The determination of the virial coefficients is of immediate relevance to cold atom experiments that study the dynamics of large fermionic clouds under low-dimensional confinement. The formalism developed in Secs. [II][V] of this paper can be extended fairly straightforwardly to three-boson and unequal-mass systems.

\section{ACKNOWLEDGEMENT}

We gratefully acknowledge support by the ARO and thank Krittika Goyal for contributions at the initial stage of this work. This work was additionally supported by the National Science Foundation through a grant for the Institute for Theoretical Atomic, Molecular and Optical Physics at Harvard University and Smithsonian Astrophysical Observatory.

\section{Appendix A: Calculation of integrals involved}

This appendix presents the evaluation of the integrals defined in Secs. III and IV The integrals $I_{n_{\rho}, n_{\rho}^{\prime}, m}^{\text {c }}(j)$ and $I_{n_{z}, n_{z}^{\prime}}^{\mathrm{p}}(j)$ are energy-independent. They are tabulated once and then used for each three-body energy considered. To evaluate the integral $I_{n_{\rho}, n_{\rho}^{\prime}, m}^{\mathrm{c}}(j)$, Eq. (31), we expand each of the three radial two-dimensional harmonic oscillator functions $R$ [see Eq. (12)] in terms of a finite sum, i.e., we use the series expansion of the associated Laguerre polynomials [47],

$$
L_{n}^{(k)}(x)=\sum_{j=0}^{n}(-1)^{j} \frac{(n+k) !}{(n-j) !(k+j) ! j !} x^{j} .
$$

$I_{n_{\rho}, n_{\rho}^{\prime}, m}^{\mathrm{c}}(j)$ then becomes a finite triple sum that contains integrals of the form

$$
\int_{0}^{\infty} \exp \left(-\frac{\eta \rho^{2}}{a_{z}^{2}}\right)\left(\frac{\rho}{a_{z}}\right)^{k} d \rho=\frac{1}{2}\left(\frac{1}{a_{z}}\right)^{k}\left(\frac{\eta}{a_{z}^{2}}\right)^{-\frac{1+k}{2}} \Gamma\left(\frac{1+k}{2}\right) .
$$

The finite sum is readily evaluated and $I_{n_{\rho}, n_{\rho}^{\prime}, m}^{\mathrm{c}}(j)$ is stored for each $n_{\rho}, n_{\rho}^{\prime}, m$ and $j$ combination. To evaluate $I_{n_{z}, n_{z}^{\prime}}^{\mathrm{p}}(j)$, Eq. (39), we rewrite each of the three one-dimensional harmonic oscillator functions $\varphi$ [see Eq. (11)] in terms of associated Laguerre polynomials instead of Hermite polynomials [48],

$$
H_{2 n}\left(z / a_{z}\right)=(-1)^{n} 2^{2 n} n ! L_{n}^{(-1 / 2)}\left(z^{2} / a_{z}^{2}\right)
$$

and

$$
H_{2 n+1}\left(z / a_{z}\right)=(-1)^{n} 2^{2 n+1} n !\left(z / a_{z}\right) L_{n}^{(1 / 2)}\left(z^{2} / a_{z}^{2}\right) .
$$

The evaluation of $I_{n_{z}, n_{z}^{\prime}}^{\mathrm{p}}(j)$ then proceeds analogously to that of $I_{n_{\rho}, n_{\rho}^{\prime}, m}^{\mathrm{c}}(j)$.

The integrals $I_{n_{z}, n_{z}^{\prime}}^{\mathrm{c}}\left(\epsilon_{\boldsymbol{\lambda}^{\prime}}, j\right)$ and $I_{n_{\rho}, n_{\rho}^{\prime}, m}^{\mathrm{p}}\left(\epsilon_{\boldsymbol{\lambda}^{\prime}}, j\right)$, Eqs. (30) and (40), are energy-dependent. To evaluate these integrals, we first "separate out" the energy dependence and then proceed along the lines discussed above. The energy dependence of $I_{n_{z}, n_{z}^{\prime}}^{\mathrm{c}}\left(\epsilon_{\boldsymbol{\lambda}^{\prime}}, j\right)$ enters through $\mathcal{G}^{1 \mathrm{D}}\left(\left[\epsilon_{\boldsymbol{\lambda}^{\prime}}-2 \eta j+1 / 2\right] E_{z} ; \sqrt{3} / 2 z ; 0\right)$. Using the identity [49]

$$
\Gamma(a) U(a, 1 / 2, x)=\sum_{k=0}^{\infty} \frac{L_{k}^{(-1 / 2)}(x)}{k+a},
$$



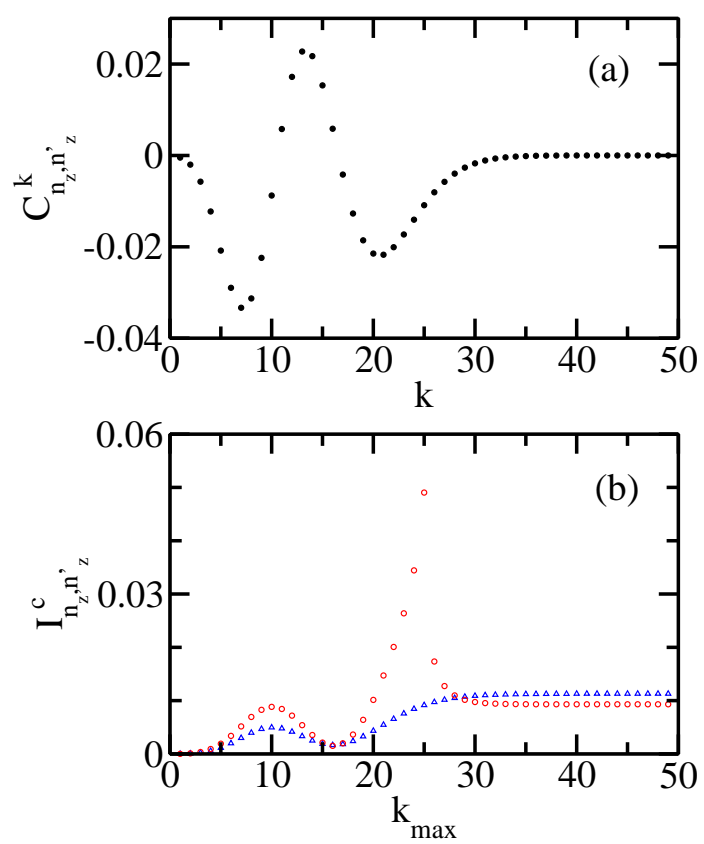

FIG. 13: Convergence study. (a) The dots show the coefficient $C_{n_{z}, n_{z}^{\prime}}^{k}$, Eq. (A7), for $n_{z}=2$ and $n_{z}^{\prime}=80$ as a function of $k$. (b) The circles and triangles show the sum $I_{n_{z}, n_{z}^{\prime}}^{\text {c }}\left(\epsilon_{\boldsymbol{\lambda}^{\prime}}, j\right)$, Eq. (A6), for $\left(\epsilon_{\boldsymbol{\lambda}^{\prime}}-2 \eta j\right) / 2=25.745$ and 40.234 , respectively, as a function of the cutoff $k_{\max }$. As in panel (a), we used $n_{z}=2$ and $n_{z}^{\prime}=80$.

$I_{n_{z}, n_{z}^{\prime}}^{\mathrm{c}}\left(\epsilon_{\boldsymbol{\lambda}^{\prime}}, j\right)$ can be written as an infinite series,

$$
I_{n_{z}, n_{z}^{\prime}}^{\mathrm{c}}\left(\epsilon_{\boldsymbol{\lambda}^{\prime}}, j\right)=\lim _{k_{\max } \rightarrow \infty} \frac{1}{2 \sqrt{2}} \sum_{k=0}^{k_{\max }} \frac{1}{k-\frac{\epsilon_{\boldsymbol{\lambda}^{\prime}}-2 \eta j}{2}} C_{n_{z}, n_{z}^{\prime}}^{k},
$$

where the coefficients $C_{n_{z}, n_{z}^{\prime}}^{k}$ are energy-independent,

$$
C_{n_{z}, n_{z}^{\prime}}^{k}=\int_{-\infty}^{\infty} \varphi_{n_{z}}(z) \varphi_{n_{z}^{\prime}}(-z / 2) \exp \left(-\frac{3 z^{2}}{8 a_{z}^{2}}\right) L_{k}^{(-1 / 2)}\left(\frac{3 z^{2}}{4 a_{z}^{2}}\right) d z
$$

The evaluation of the $C_{n_{z}, n_{z}^{\prime}}^{k}$ now proceeds as above.

The series introduced in Eq. (A6) diverges if $\left(\epsilon_{\boldsymbol{\lambda}^{\prime}}-2 \eta j\right) / 2$ is a positive integer or zero, that is, in the non-interacting limit. In practice, this does not pose a constraint since the energy grid can be chosen such that it does not contain the non-interacting energies. To analyze the dependence of $I_{n_{z}, n_{z}^{\prime}}^{\mathrm{c}}\left(\epsilon_{\boldsymbol{\lambda}^{\prime}}, j\right)$ on the upper summation index $k_{\max }$, Fig. 13)(a) shows the behavior of $C_{n_{z}, n_{z}^{\prime}}^{k}$ for fixed $n_{z}$ and $n_{z}^{\prime}$ as a function of $k$. In this example, $C_{n_{z}, n_{z}^{\prime}}^{k}$ vanishes nearly identically for $k \gtrsim 30$. The coefficients $C_{n_{z}, n_{z}^{\prime}}^{k}$ are multiplied by the $k$-dependent prefactor $\left[k-\left(\epsilon_{\boldsymbol{\lambda}^{\prime}}-2 \eta j\right) / 2\right]^{-1}$. This prefactor is maximal for $k \approx\left(\epsilon_{\boldsymbol{\lambda}^{\prime}}-2 \eta j\right) / 2$. Figure 13(b) shows the quantity $I_{n_{z}, n_{z}^{\prime}}^{\mathrm{c}}\left(\epsilon_{\boldsymbol{\lambda}^{\prime}}, j\right)$ as a function of $k_{\max }$ for the same $n_{z}$ and $n_{z}^{\prime}$ as in Fig. 13(a) but two different values of $\left(\epsilon_{\boldsymbol{\lambda}^{\prime}}-2 \eta j\right) / 2$, that is, for $\left(\epsilon_{\boldsymbol{\lambda}^{\prime}}-2 \eta j\right) / 2=25.745$ and 40.234 . In the first case, the absolute value of the prefactor takes its maximum at $k=26$, where the coefficients $C_{n_{z}, n_{z}^{\prime}}^{k}$ are non-zero. Correspondingly, $I_{n_{z}, n_{z}^{\prime}}^{\mathrm{c}}$ shows a sharp peak near $k_{\max }=26$ and then smoothly approaches its asymptotic value [see circles in Fig. [13(b)]. In the second case, the absolute value of the prefactor takes its maximum at $k=40$, where the $C_{n_{z}, n_{z}^{\prime}}^{k}$ coefficients are very small. Correspondingly, the $C_{n_{z}, n_{z}^{\prime}}^{k}$ coefficients suppress the maximum of the prefactor and the quantity $I_{n_{z}, n_{z}^{\prime}}^{\mathrm{c}}$ approaches its asymptotic value for $k_{\max } \approx 30$ [see triangles in Fig. 13(b)]. We find that the choice of $k_{\max } \gtrsim 2 \max \left(n_{z}, n_{z}^{\prime}\right)$ yields well converged values for $I_{n_{z}, n_{z}^{\prime}}^{\mathrm{c}}\left(\epsilon_{\boldsymbol{\lambda}^{\prime}}, j\right)$ for all energies considered.

In an alternative approach, we determined $I_{n_{z}, n_{z}^{\prime}}^{\mathrm{c}}\left(\epsilon_{\boldsymbol{\lambda}^{\prime}}, j\right)$ through numerical integration for each $n_{z}, n_{z}^{\prime}, n_{\rho}^{\prime}, m, j$ and energy. We have found this approach to be computationally more time-consuming than the tabulation approach discussed above. 
The evaluation of the integral $I_{n_{\rho}, n_{\rho}^{\prime}, m}^{\mathrm{p}}\left(\epsilon_{\boldsymbol{\lambda}^{\prime}}, j\right)$, Eq. (40), proceeds analogously to that of $I_{n_{z}, n_{z}^{\prime}}^{\mathrm{c}}\left(\epsilon_{\boldsymbol{\lambda}^{\prime}}, j\right)$. We use [49]

$$
\Gamma(a) U(a, 1, x)=\sum_{k=0}^{\infty} \frac{L_{k}(x)}{k+a}
$$

to separate out the energy-dependence that enters through $\mathcal{G}^{2 \mathrm{D}}$. The integral is then written as

$$
I_{n_{\rho}, n_{\rho}^{\prime}, m}^{\mathrm{p}}\left(\epsilon_{\boldsymbol{\lambda}^{\prime}}, j\right)=\lim _{k_{\max } \rightarrow \infty} \frac{1}{2 \pi} \sum_{k=0}^{k_{\max }} \frac{1}{k-\frac{\epsilon_{\boldsymbol{\lambda}^{\prime}}-2 j}{2 \eta}} C_{n_{\rho}, n_{\rho}^{\prime}, m}^{k},
$$

where

$$
C_{n_{\rho}, n_{\rho}^{\prime}, m}^{k}=\int_{0}^{\infty} R_{n_{\rho}, m}(\rho) R_{n_{\rho}^{\prime}, m}\left(\frac{\rho}{2}\right) \exp \left(-\frac{3 \eta \rho^{2}}{8 a_{z}^{2}}\right) L_{k}\left(\frac{3 \eta \rho^{2}}{4 a_{z}^{2}}\right) \rho d \rho .
$$

We tabulate $C_{n_{\rho}, n_{\rho}^{\prime}, m}^{k}$ and calculate $I_{n_{\rho}, n_{\rho}^{\prime}, m}^{\mathrm{p}}\left(\epsilon_{\boldsymbol{\lambda}^{\prime}}, j\right)$ for each $\epsilon_{\boldsymbol{\lambda}^{\prime}}$ "on the fly". The convergence behavior of $I_{n_{\rho}, n_{\rho}^{\prime}, m}^{\mathrm{p}}\left(\epsilon_{\boldsymbol{\lambda}^{\prime}}, j\right)$ is similar to that of $I_{n_{z}, n_{z}^{\prime}}^{\mathrm{c}}\left(\epsilon_{\boldsymbol{\lambda}^{\prime}}, j\right)$.

[1] D. Blume, Rep. Prog. Phys. 75, 046401 (2012).

[2] S. Giorgini, L. P. Pitaevskii, and S. Stringari, Rev. Mod. Phys. 80, 1215 (2008).

[3] I. Bloch, J. Dalibard, and W. Zwerger, Rev. Mod. Phys. 80, 885 (2008).

[4] C. Chin, R. Grimm, P. Julienne, and E. Tiesinga, Rev. Mod. Phys. 82, 1225 (2010).

[5] T. Busch, B.-G. Englert, K. Rzążewski, and M. Wilkens, Found. Phys. 28, 549 (1998).

[6] Z. Idziaszek and T. Calarco, Phys. Rev. A 71, 050701(R) (2005).

[7] Z. Idziaszek and T. Calarco, Phys. Rev. A 74, 022712 (2006).

[8] J. P. Kestner and L.-M. Duan, Phys. Rev. A 76, 033611 (2007).

[9] D. Blume, J. von Stecher, and C. H. Greene, Phys. Rev. Lett. 99, 233201 (2007).

[10] J. von Stecher and C. H. Greene, Phys. Rev. Lett. 99, 090402 (2007).

[11] T.-L. Ho and E. J. Mueller, Phys. Rev. Lett. 92, 160404 (2004).

[12] T.-L. Ho, Phys. Rev. Lett. 92, 090402 (2004).

[13] G. Rupak, Phys. Rev. Lett. 98, 090403 (2007).

[14] X.-J. Liu, H. Hu, and P. D. Drummond, Phys. Rev. Lett. 102, 160401 (2009).

[15] X.-J. Liu, H. Hu, and P. D. Drummond, Phys. Rev. A 82, 023619 (2010).

[16] X.-J. Liu, H. Hu, and P. D. Drummond, Phys. Rev. B 82, 054524 (2010).

[17] S. Nascimbène, N. Navon, K. J. Jiang, F. Chevy, and C. Salomon, Nature 463, 1057 (2010).

[18] M. J. H. Ku, A. T. Sommer, L. W. Cheuk, and M. W. Zwierlein, Science 335, 563 (2012).

[19] K. M. Daily, and D. Blume, Phys. Rev. A 85, 013609 (2012).

[20] M. Olshanii, Phys. Rev. Lett. 81, 938 (1998).

[21] T. Bergeman, M. G. Moore, and M. Olshanii, Phys. Rev. Lett. 91, 163201 (2003).

[22] D. S. Petrov and G. V. Shlyapnikov, Phys. Rev. A 64, 012706 (2001).

[23] H. Moritz, T. Stöferle, K. Günter, M. Köhl, and T. Esslinger, Phys. Rev. Lett. 94, 210401 (2005).

[24] E. Fermi, Nuovo Cimento 11, 157 (1934).

[25] K. Huang, Statistical Mechanics. John Wiley \& Sons, Inc (1963).

[26] K. Huang and C. N. Yang, Phys. Rev. 105, 767 (1957).

[27] D. Blume and C. H. Greene, Phys. Rev. A 65, 043613 (2002).

[28] E. L. Bolda, E. Tiesinga, and P. S. Julienne, Phys. Rev. A 66, 013403 (2002).

[29] E. Nielsen, D. V. Fedorov, A. S. Jensen, and E. Garrido, Phys. Rep. 347, 373 (2001).

[30] O. I. Kartavtsev and A. V. Malykh, J. Phys. B 40, 1429 (2007).

[31] S. T. Rittenhouse, N. P. Mehta, and C. H. Greene, Phys. Rev. A 82, 022706 (2010).

[32] C. Mora, R. Egger, A. O. Gogolin, and A. Komnik, Phys. Rev. Lett. 93, 170403 (2004).

[33] C. Mora, R. Egger, and A. O. Gogolin, Phys. Rev. A 71, 052705 (2005).

[34] D. S. Petrov, Phys. Rev. A 67, 010703(R) (2003).

[35] F. Werner and Y. Castin, Phys. Rev. Lett. 97, 150401 (2006).

[36] A. T. Sommer, L. W. Cheuk, M. J. H. Ku, W. S. Bakr, and M. W. Zwierlein, Phys. Rev. Lett. 108, 045302 (2012).

[37] M. Koschorreck, D. Pertot, E. Vogt, B. Fröhlich, M. Feld, and M. Köhl, Nature 485, 619 (2012).

[38] Y. Zhang, W. Ong, I. Arakelyan, and J. E. Thomas, Phys. Rev. Lett. 108, 235302 (2012).

[39] E. Braaten and H.-W. Hammer, Phys. Rep. 428, 259 (2006).

[40] Y. Nishida and S. Tan, Few-Body Systems 51, 191 (2011). 
[41] Our notation follows that employed in Ref. [19] but differs from that employed in Ref. [14]. In Ref. 14], the secondand third-order virial coefficients for the isotropically trapped system are denoted by $\Delta b_{2}$ and $\Delta b_{3}$, respectively. The relationship between our $b_{n}$ and the $\Delta b_{n}$ of Ref. [14] for an isotropic trap is $\Delta b_{2}=b_{2} / 2$ and $\Delta b_{3}=b_{3} / 2$.

[42] S.-G. Peng, X.-J. Liu, H. Hu, and S.-Q. Li, Phys. Lett. A 375, 2979 (2011).

[43] C. Menotti, P. Pedri, and S. Stringari, Phys. Rev. Lett. 89, 250402 (2002).

[44] The second term on the right hand side of Eq. (24) in Ref. [42] is off by a factor of $1 / \sqrt{2}$; to see this one has to realize that Ref. 42] defines the oscillator length in terms of the atom mass while we use the reduced mass.

[45] T. Lompe, T. B. Ottenstein, F. Serwane, A. N. Wenz, G. Zürn, and S. Jochim. Science 330, 940 (2010).

[46] G. Zürn, F. Serwane, T. Lompe, A. N. Wenz, M. G. Ries, J. E. Bohn, and S. Jochim. Phys. Rev. Lett. 108, 075303 (2012).

[47] Handbook of Mathematical Functions, edited by M. Abramowitz and I. E. Stegun, 10th ed. (Department of Commerce, Washington, DC, 1972).

[48] Entries 22.5.40 and 22.5.41 of Ref. [47].

[49] K. Kanjilal, J. L. Bohn, and D. Blume, Phys. Rev. A 75, 052705 (2007). 